\title{
Advanced Tertiary Lymphoid Tissues in Protocol Biopsies are Associated with Progressive Graft Dysfunction in Kidney Transplant Recipients
}

\section{$\operatorname{AUTHOR}(\mathrm{S})$ :}

Lee, Yu Ho; Sato, Yuki; Saito, Mitsuru; Fukuma, Shingo; Saito, Masaya; Yamamoto, Shigenori; Komatsuda, Atsushi; ... Habuchi, Tomonori; Floege, Juergen; Yanagita, Motoko

\section{CITATION:}

Lee, Yu Ho ...[et al]. Advanced Tertiary Lymphoid Tissues in Protocol Biopsies are Associated with Progressive Graft Dysfunction in Kidney Transplant Recipients. Journal of the American Society of Nephrology 2022, 33(1): 186-200

\section{ISSUE DATE:}

2022-01

URL:

http://hdl.handle.net/2433/267192

\section{RIGHT:}

This is not the published version. Please cite only the published version. この論文は出版社版でありません。引用の際には出版社版をご確認ご 利用ください。 


\section{JASN}

\section{Advanced Tertiary Lymphoid Tissues in Protocol Biopsies are Associated with Progressive Graft Dysfunction in Kidney Transplant Recipients}

\begin{tabular}{|c|c|}
\hline Journal: & Journal of the American Society of Nephrology \\
\hline Manuscript ID & JASN-2021-05-0715.R1 \\
\hline Manuscript Type: & Original Article - Clinical Research \\
\hline $\begin{array}{r}\text { Date Submitted by the } \\
\text { Author: }\end{array}$ & 25-Aug-2021 \\
\hline Complete List of Authors: & $\begin{array}{l}\text { Lee, Yu Ho; Kyoto University Graduate School of Medicine Faculty of } \\
\text { Medicine, Department of Nephrology; CHA Bundang Medical Center, } \\
\text { Division of Nephrology, Department of Internal Medicine } \\
\text { Sato, Yuki; Kyoto University Graduate School of Medicine Faculty of } \\
\text { Medicine, Department of Nephrology; Kyoto University Graduate School } \\
\text { of Medicine Faculty of Medicine, Medical Innovation Center TMK Project } \\
\text { Saito, Mitsuru; Akita University Graduate School of Medicine School of } \\
\text { Medicine, Department of Urology } \\
\text { Fukuma, Shingo; Kyoto University Graduate School of Medicine Faculty } \\
\text { of Medicine, Human Health Sciences } \\
\text { Saito, Masaya; Akita University Graduate School of Medicine School of } \\
\text { Medicine, Department of Hematology, Nephrology, and Rheumatology } \\
\text { Yamamoto, Shigenori; Kyoto University Graduate School of Medicine } \\
\text { Faculty of Medicine, Department of Nephrology; Kyoto University } \\
\text { Graduate School of Medicine Faculty of Medicine, Medical Innovation } \\
\text { Center TMK Project } \\
\text { Komatsuda, Atsushi; Akita University Graduate School of Medicine } \\
\text { School of Medicine, Department of Hematology, Nephrology, and } \\
\text { Rheumatology } \\
\text { Fujiyama, Nobuhiro; Akita University Hospital, Center for Kidney Disease } \\
\text { and Transplantation } \\
\text { Satoh, Shigeru; Akita University Hospital, Center for Kidney Disease and } \\
\text { Transplantation } \\
\text { Lee, Sang-Ho; Kyung Hee University, Division of Nephrology, } \\
\text { Department of Internal Medicine } \\
\text { Boor, Peter; RWTH Aachen University, Institute of Pathology, } \\
\text { Department of Nephrology, \& Electron Microscopy Facility } \\
\text { Habuchi, Tomonori; Akita University Graduate School of Medicine School } \\
\text { of Medicine, Department of Urology } \\
\text { Floege, Juergen; RWTH Aachen University, Division of Nephrology } \\
\text { Yanagita, Motoko; Kyoto University Graduate School of Medicine Faculty } \\
\text { of Medicine, Department of Nephrology; Kyoto University Institute for } \\
\text { the Advanced Study of Human Biology }\end{array}$ \\
\hline Keywords: & $\begin{array}{l}\text { kidney transplantation, renal biopsy, transplant outcomes, Renal } \\
\text { pathology, Biopsy, Lymphoid Tissue }\end{array}$ \\
\hline
\end{tabular}


Authors: Lee, Yu Ho; Sato, Yuki; Saito, Mitsuru; Fukuma, Shingo; Saito, Masaya; Yamamoto, Shigenori; Komatsuda, Atsushi; Fujiyama, Nobuhiro; Satoh, Shigeru; Lee, Sang-Ho; Boor, Peter; Habuchi, Tomonori; Floege, Juergen; Yanagita, Motoko

Title: Advanced Tertiary Lymphoid Tissues in Protocol Biopsies are Associated with Progressive Graft Dysfunction in Kidney Transplant Recipients

Running title: Tertiary lymphoid tissues in transplanted kidneys

Manuscript Type: Original Article - Clinical Research

Manuscript Category: Clinical transplantation

Funders: Bundesministerium fã $1 / 4$ r Forschung und Technologie, (Grant / Award Number: 'STOP-FSGS01GM1518A')

Deutsche Forschungsgemeinschaft, (Grant / Award Number: 'BO3755/3-1 ','BO3755/61','SFB/TRR219','SFB/TRR57 ')

Japan Agency for Medical Research and Development, (Grant / Award Number: '21gm1210009','21gm5010002','21/m02030','JP19gm0610011','TR-SPRINT')

Japan Society for the Promotion of Science, (Grant / Award Number: '17H05642','18H04673','26293202')

Ministry of Education, Culture, Sports, Science and Technology, (Grant / Award Number: 'World Premier International Research Center Initia')

National Research Foundation of Korea, (Grant / Award Number: 'NRF-2021R1G1A1014115')

RWTH Interdisciplinary Centre for Clinical Research, (Grant / Award Number: 'IZKF: O3-7')

Sumitomo Foundation, (Grant / Award Number: )

Takeda Science Foundation, (Grant / Award Number: )

Uehara Memorial Foundation, (Grant / Award Number: )

Financial Disclosure: CUST_FINANCIAL_DISCLOSURE :No data available. YS is employed by TMK project. MY receives research grants from Astellas, Chugai, Daiichi Sankyo, Fujiyakuhin, Kyowa Hakko Kirin, Mitsubishi Tanabe, MSD, Nippon Boehringer Ingelheim, and Torii; Honoraria from Astellas, Kyowa Kirin, Chugai, and others for lecture honoraria; and Other Interests/Relationships with Japanese Society of Nephrology, and International Society of Nephrology. S. Fukuma reports Consultancy Agreements with Kyowa Hakko Kirin, Rege Nephro; Research Funding from Kyowa Hakko Kirin, CANCER SCAN; Honoraria from Kyowa Hakko Kirin; and Scientific Advisor or Membership with Kyowa Hakko Kirin. S. Yamamoto reports Other Interests/Relationships with the Japanese society of Nephrology, and The Japanese society for Dialysis Therapy. T. Habuchi reports Research Funding from Kyowa Kirin Co., Astellas Pharma Inc., Bayer AG., Chugai Pharmaceutical Co.; and Honoraria from Kyowa Kirin Co., Astellas Pharma Inc., Bayer AG., Chugai Pharmaceutical Co. J. Floege reports Consultancy Agreements with Calliditas, Travere, Novartis, Idorsia, Omeros, Bayer, AstraZeneca; and Honoraria from Calliditas, Travere, Novartis, Idorsia, Omeros, Bayer, AstraZeneca, Vifor, and Fresenius. All remaining authors report no conflicts of interest. 
Study Group/Organization Name: CUST_STUDY_GROUP/ORGANIZATION_NAME :No data available.

Study Group Members' Names: CUST_STUDY_GROUP_MEMBERS :No data available.

Total number of words: 3616

Abstract: $<$ b $>$ Background: $</ b>$ Tertiary lymphoid tissues (TLTs) are ectopic lymphoid tissues found in chronically inflamed organs. Although studies have documented TLT formation in transplanted kidneys, the clinical relevance of these TLTs remains controversial. We examined the impacts of TLTs on future graft function using our histological TLT maturity stages and the association between TLTs and Banff pathologic scores. We also analyzed the risk factors for the development of TLTs $<$ b $>$ Methods: $</$ b $>$ Serial protocol biopsy samples (0-hour, 1-, 6-, and 12-months) without rejection were retrospectively analyzed from 214 patients who underwent living donor kidney transplantation. TLTs were defined as lymphocyte aggregates with signs of proliferation and their stages were determined by the absence (stage I) or presence (stage II) of follicular dendritic cells.

$\langle$ b $>$ Results: $</ b>$ Only $4 \%$ of patients exhibited TLTs at the 0 -hour biopsy. Prevalence increased to almost $50 \%$ at the 1-month biopsy and then slightly further for 12 months. The proportion of advanced stage II TLTs increased gradually, reaching $19 \%$ at the 12-month biopsy. Presence of stage II TLTs was associated with higher risk of renal function decline after transplantation compared to patients with no TLT or stage I TLTs. Stage II TLTs were associated with more severe tubulitis and interstitial fibrosis/tubular atrophy at 12 months and predicted poorer graft function independently from the degree of interstitial inflammation. Pre-transplantation rituximab treatment dramatically attenuated the development of stage II TLTs.

$\langle\mathrm{b}\rangle$ Conclusions: $</ \mathrm{b}\rangle$ TLTs are commonly found in clinically stable transplanted kidneys. Advanced stage II TLTs are associated with progressive graft dysfunction, independent of interstitial inflammation 
Copyright 2021 by ASN, Published Ahead of Print on 11/1/21, Accepted/Unedited Version

\section{Significance Statement}

Tertiary lymphoid tissues (TLTs) are frequently found in transplanted kidneys, but their prevalence and clinical significance remain uncertain. Serial protocol kidney transplant biopsies without signs of rejection were collected and TLTs staged according to the presence of proliferating lymphocytes and follicular dendritic cells. TLTs rapidly developed within 1 month after kidney transplantation in approximately half the 214 patients. Advanced TLTs, defined as the presence of follicular dendritic cells, was associated with progressive decline in graft function independent of interstitial inflammation score. These findings suggest that advanced TLTs are strongly associated with late graft dysfunction even in the absence of rejection. 
Copyright 2021 by ASN, Published Ahead of Print on 11/1/21, Accepted/Unedited Version

\section{Advanced Tertiary Lymphoid Tissues in Protocol Biopsies are Associated with Progressive Graft Dysfunction in Kidney Transplant Recipients}

Yu Ho Lee ${ }^{1,2,13}$, Yuki Sato ${ }^{1,3,13}$, Mitsuru Saito ${ }^{4}$, Shingo Fukuma ${ }^{5}$, Masaya Saito ${ }^{6}$, Shigenori Yamamoto $^{1,3}$, Atsushi Komatsuda ${ }^{6}$, Nobuhiro Fujiyama ${ }^{7}$, Shigeru Satoh ${ }^{7}$, Sang-Ho Lee ${ }^{8}$, Peter Boor ${ }^{9,10,11}$, Tomonori Habuchi ${ }^{4}$, Jürgen Floege ${ }^{10}$, and Motoko Yanagita ${ }^{1,12}$

${ }^{1}$ Department of Nephrology, ${ }^{3}$ Medical Innovation Center TMK Project, ${ }^{5}$ Human Health Sciences, Graduate School of Medicine, Kyoto University, Kyoto, Japan

${ }^{2}$ Division of Nephrology, Department of Internal Medicine, CHA Bundang Medical Center, CHA University, Seongnam, Republic of Korea

${ }^{4}$ Department of Urology, ${ }^{6}$ Department of Hematology, Nephrology, and Rheumatology, Graduate School of Medicine, Akita University, Akita, Japan

${ }^{7}$ Center for Kidney Disease and Transplantation, Akita University Hospital, Akita, Japan ${ }^{8}$ Division of Nephrology, Department of Internal Medicine, Kyung Hee University, Seoul, Republic of Korea

${ }^{9}$ Institute of Pathology, ${ }^{10}$ Division of Nephrology, ${ }^{11}$ Electron Microscopy Facility, RWTH University of Aachen, Germany

${ }^{12}$ Institute for the Advanced Study of Human Biology (ASHBi), Kyoto University, Kyoto, Japan

${ }^{13}$ Y.H.L. and Y.S. contributed equally to this work.

Short running title: Tertiary lymphoid tissues in transplanted kidneys

Word count for abstract: 251 text: 3616 (including significance statement and abstract)

\section{To whom correspondence should be addressed}

Motoko Yanagita, Department of Nephrology, Graduate School of Medicine, Kyoto University, Shogoin-Kawahara-cho 54, Sakyo-ku, Kyoto 606-8507, Japan

(Tel)+81-75-751-3860, (Fax)+81-75-751-3859, (E-mail) motoy@kuhp.kyoto-u.ac.jp

Keywords: kidney transplantation, tertiary lymphoid tissue, protocol biopsy, long term graft function,

rituximab 
Copyright 2021 by ASN, Published Ahead of Print on 11/1/21, Accepted/Unedited Version

\begin{abstract}
Background: Tertiary lymphoid tissues (TLTs) are ectopic lymphoid tissues found in chronically inflamed organs. Although studies have documented TLT formation in transplanted kidneys, the clinical relevance of these TLTs remains controversial. We examined the impacts of TLTs on future graft function using our histological TLT maturity stages and the association between TLTs and Banff pathologic scores. We also analyzed the risk factors for the development of TLTs.
\end{abstract}

Methods: Serial protocol biopsy samples (0-hour, 1-, 6-, and 12-months) without rejection were retrospectively analyzed from 214 patients who underwent living donor kidney transplantation. TLTs were defined as lymphocyte aggregates with signs of proliferation and their stages were determined by the absence (stage I) or presence (stage II) of follicular dendritic cells.

Results: Only $4 \%$ of patients exhibited TLTs at the 0 -hour biopsy. Prevalence increased to almost $50 \%$ at the 1-month biopsy and then slightly further for 12 months. The proportion of advanced stage II TLTs increased gradually, reaching 19\% at the 12-month biopsy. Presence of stage II TLTs was associated with higher risk of renal function decline after transplantation compared to patients with no TLT or stage I TLTs. Stage II TLTs were associated with more severe tubulitis and interstitial fibrosis/tubular atrophy at 12 months and predicted poorer graft function independently from the degree of interstitial inflammation. Pre-transplantation rituximab treatment dramatically attenuated the development of stage II TLTs.

Conclusions: TLTs are commonly found in clinically stable transplanted kidneys. Advanced stage II TLTs are associated with progressive graft dysfunction, independent of interstitial inflammation. 
Copyright 2021 by ASN, Published Ahead of Print on 11/1/21, Accepted/Unedited Version

\section{Introduction}

Kidney transplantation is an ideal treatment for patients with end-stage renal disease. Whereas short-term graft survival had been greatly improved in the last three decades, long term graft survival has changed only marginally ${ }^{1,2}$. Among various factors affecting the outcomes of transplanted kidneys, chronic intra-graft inflammation has been considered one of the most important components that contribute to persistent allograft injury ${ }^{3-6}$. Studies have consistently suggested that subclinical rejection frequently leads to deterioration of transplanted kidneys if left untreated ${ }^{7-10}$. Even mild tubulointerstitial inflammation was associated with poor graft outcomes after kidney transplantation, highlighting the relationship between unresolved inflammation and progressive functional decline ${ }^{11}$. For these reasons, better understanding and proper management of graft inflammation is a prerequisite to longterm graft survival.

Persistent inflammatory stimuli often give rise to the development of tertiary lymphoid tissues (TLTs), i.e., inducible ectopic lymphoid tissues that arise in chronic inflammatory conditions such as aging, cancer, autoimmune diseases and in transplanted organs ${ }^{12-15} . \mathrm{T}$ and B lymphocytes are the main hematopoietic components of TLTs, and specialized fibroblasts provide structural support and produce homeostatic chemokines such as CXCL13 $3^{16-22}$. Although the functional roles of TLTs are context-dependent, we have previously described a strong association between renal TLTs and maladaptive repair in rodent models ${ }^{20}$.

To provide objective and standardized analytic methodology, we recently proposed a new TLT staging strategy based on the presence of follicular dendritic cells (FDCs) and germinal centers, both of which represent cellular components of advanced TLTs ${ }^{23}$. TLT stages 
Copyright 2021 by ASN, Published Ahead of Print on 11/1/21, Accepted/Unedited Version

positively correlated with the severity of kidney injury and inflammation, suggesting the potential to serve as additional histological markers of tissue inflammation.

The presence of TLTs in transplanted kidneys is well documented ${ }^{24-35}$. Nevertheless, their clinical relevance remains controversial. The main reasons for these conflicting results include that TLTs were not separated from concurrent rejection, and the definition of TLTs has been inconsistent across studies ${ }^{14,36-38}$. The facts that TLTs were frequently observed both in rejected and tolerated murine allografts further complicate their functional identity in transplanted kidneys ${ }^{39-41}$. To overcome these issues and to clarify the impacts of TLTs on graft functions, we utilized two major strategies. First, we collected protocol biopsy samples from kidney transplant recipients without overt evidence of rejection to directly investigate the relationship between TLTs and graft function. Second, using our recently established TLT staging method ${ }^{23}$, we classified TLTs based on their phenotypes, and analyzed the association between TLT stages and graft outcomes. Here, we demonstrate that TLTs developed in almost half of clinically stable patients and that the presence of advanced stage II TLTs was associated with progressive functional decline of renal allograft in comparison with stable graft function in patients without TLTs or with stage I TLTs. 
Copyright 2021 by ASN, Published Ahead of Print on 11/1/21, Accepted/Unedited Version

\section{Methods}

\section{Study population and protocol biopsy sample acquisition}

An overview of the study design and patient recruitment strategy is given in Figure 1. We retrospectively screened 241 patients who underwent their first living donor kidney transplantation between July 2004 and December 2016 at Akita University in Japan. Four serial protocol biopsies were obtained from each patient during this period. A 0-hour protocol biopsy was performed during cold-saline perfusion after kidney explanation from the donor. Subsequently, recipients underwent protocol biopsies at 1 month, 6 months, and 12 months after transplantation. At least two cores of graft tissue were obtained at each biopsy, paraffinembedded, and subjected to conventional histologic stains and immunofluorescence. Patients were excluded if they met one or more of the following criteria: 1) occurrence of biopsyproven acute rejection within the first year of transplantation; 2) occurrence of BK virusassociated nephropathy within the first year of transplantation; 3) non-recovery of renal allograft function $\left(<30 \mathrm{ml} / \mathrm{min} / 1.73 \mathrm{~m}^{2}\right)$ over the first year of transplantation; or 4$)$ loss to follow-up within the first year of transplantation. To be specific, recipients with subclinical rejection (biopsy-proven acute $\mathrm{T}$ cell-mediated or antibody-mediated rejection without an elevation in serum creatinine level) were excluded, while those with borderline $\mathrm{T}$ cellmediated rejection $(\mathrm{t}>0$ with $\mathrm{i} 0$ or $\mathrm{i} 1$, or $\mathrm{t} 1$ with $\mathrm{i} 2$ or $\mathrm{i} 3$ without an elevation in serum creatinine levels) were included in this study ${ }^{42-44}$.

Information regarding the baseline characteristics of the recipients and donors was obtained at the time of kidney transplantation and during visits to the outpatient clinic. Donor specific antibody was retrospectively measured in the sera at 1-year after transplantation stored until use using Luminex-based SAB kits (LABSCreen ${ }^{\circledR}$ PRA and LABSCreen ${ }^{\circledR}$ Single Antigen, Thermo Scientific, Waltham, MA). Positive evaluations were made as previously described ${ }^{45}$. Indication biopsy was performed when patients had an unexplained rise in serum creatinine 
Copyright 2021 by ASN, Published Ahead of Print on 11/1/21, Accepted/Unedited Version

( $>25 \%$ from baseline value) during the follow-up period, but the presence of TLTs in these samples was not assessed in this study. Kidney function was measured as the estimated glomerular filtration rate (eGFR) using the Chronic Kidney Disease Epidemiology Collaboration formula for the Japanese population ${ }^{46}$. All diagnoses and Banff pathologic scores were determined and rescored by a single experienced transplant nephrologist, in accordance with Banff 2017 criteria $^{42}$.

All human specimens were analyzed after informed consent, and approval of the ethics committees at Akita and Kyoto University hospitals were obtained. This study adhered to the Declaration of Istanbul.

\section{Outcome measures}

The primary endpoint was the occurrence of death-censored renal function decline, defined as a decline of at least $30 \%$ in the eGFR from 1-year post-transplant graft function. The secondary endpoint was renal allograft function after kidney transplantation.

\section{Immunosuppressive regimen}

Protocols of immunosuppressive drugs are described elsewhere ${ }^{47}$. Briefly, all patients received basiliximab (20 $\mathrm{mg}$ at day 0 and day 4) as induction immunosuppression, followed by maintenance immunosuppression with prednisolone, mycophenolate mofetil, and tacrolimus. Patients undergoing ABO-incompatible kidney transplantation received a single dose of rituximab (200 mg) 3 weeks before transplantation followed by plasma exchange or double-filtration plasmapheresis and the administration of intravenous immunoglobulin. Patients diagnosed with borderline $\mathrm{T}$ cell-mediated rejection in protocol biopsies were routinely treated with $10-20 \mathrm{mg} / \mathrm{kg}$ of intravenous methylprednisolone for $2-3$ consecutive days depending on the degree of graft inflammation and patient status, unless contraindicated. 
Copyright 2021 by ASN, Published Ahead of Print on 11/1/21, Accepted/Unedited Version

\section{Identification and evaluation of TLTs in transplanted kidneys}

In the present study, we defined TLTs as organized lymphocyte aggregates with the signs of proliferation as described previously ${ }^{23}, 48$. Because TLT sizes in transplanted kidneys were variable (Supplemental Figure 1A), we defined organized lymphocyte aggregates as more than 60 lymphocytes ( $\mathrm{T}$ cells or B cells) in this study (Supplemental Figure 1A-C). After diagnosis of TLTs, we determined stages of each TLT.

Identification and quantification of TLT stages was determined through two steps as follows: 1) identification of mononuclear cell infiltrates in permissive areas for TLT formation, which include subcapsular, periglomerular and perivascular area, with periodic acid-Schiff (PAS)stained graft sections; and 2) assessment of the lymphocyte infiltrates using immunofluorescence of (a) CD3 $\varepsilon$ and CD20, and (b) Ki67 and CD21 in two serial sections for each individual, as described previously ${ }^{23}$. After the diagnosis of TLTs, we determined TLT stages of each TLT.

TLT stages were defined as follows:

i) TLT lacking either FDC or germinal center: stage I TLT

ii) TLT containing FDC but lacking germinal center: stage II TLT

iii) TLT containing both FDC and germinal center: stage III TLT

FDCs were defined as the cells strongly positive for non-hematopoietic CD21 signals within TLTs. Germinal centers were defined with Ki67-positive cell clusters, which contained more than 15 Ki67-positive cells per cluster, in B cell areas.

\section{Renal immunofluorescence}

Immunofluorescence studies were performed on tissues from the same block as were used for the preparation of PAS-stained slides. Immunofluorescence staining of biopsy tissues was 
Copyright 2021 by ASN, Published Ahead of Print on 11/1/21, Accepted/Unedited Version performed as previously described ${ }^{49}$. The following primary antibodies were used in these experiments: anti-CD3e (catalog ab5690; Abcam, Cambridge, UK), anti-CD20 (catalog 140202; eBioscience, San Diego, CA), Ki67 (catalog ab16667; Abcam), anti-CD21 (catalog ab75985; Abcam, and catalog MA5-11417; Thermo Scientific, Waltham, MA), anti-CD45 (catalog 14-9457; eBioscience), anti-p75NTR (catalog AF1157; R\&D, Minneapolis, MN), and anti-CXCL13 (catalog AF801; R\&D). Staining was visualized using appropriate secondary antibodies. Cell nuclei were counterstained with DAPI. All immunofluorescence samples were analyzed using a confocal microscope (FV1000D; Olympus, Japan).

\section{Statistical analysis}

Statistical analyses were performed using SPSS for Windows, version 20.0 (IBM Corp., Armonk, NY) and STATA 14.1 (StataCorp, College Station, TX). Baseline patient characteristics and clinical parameters were expressed as mean \pm standard deviation (SD) or as numbers of patients and percentages. Time on dialysis was expressed as median $\left[1^{\text {st }}\right.$ and $3^{\text {rd }}$ interquartile range] because it was non-normally distributed. Temporal changes in the TLT stages were assessed by trend analysis. Renal function decline, assessed in a time-to-event analysis, was analyzed by Cox proportional-hazards models with an adjustment for age, sex, the presence of diabetes after transplantation, $\mathrm{ABO}$ incompatibility, positive crossmatch, the presence of donor specific antibody at 1-year post-transplantation, and pre-transplantation donor eGFR. To further compare the differences in repeatedly measured eGFR during the follow-up period between groups, we used a linear mixed-effect model with robust variance estimation $^{50}$, adjusting for baseline covariates including donor and recipient age and gender, recipient body mass index, preemptive kidney transplantation, the presence of diabetes after transplantation, the number of HLA mismatching, positive crossmatch, ABO incompatibility, the use of immunosuppressants, baseline graft function, the presence of donor specific 
Copyright 2021 by ASN, Published Ahead of Print on 11/1/21, Accepted/Unedited Version antibody at 1-year post-transplantation, and pre-transplantation donor eGFR. Baseline graft function was set as eGFR levels at each biopsy-time point. Logistic regression analysis was performed to identify risk factors for the development of TLTs. The relationship between the use of pre-transplantation rituximab and TLTs was analyzed using Pearson's chi-square test or Fisher's exact test as appropriate. Finally, the overall comparisons of Banff pathologic scores with TLT scores were performed using the Kruskal-Wallis test, and the MannWhitney test was used for the comparisons of each group. $P$-values less than 0.05 were considered statistically significant. 
Copyright 2021 by ASN, Published Ahead of Print on 11/1/21, Accepted/Unedited Version

\section{Results}

\section{Baseline characteristics of enrolled patients}

A total of 214 kidney transplant recipients were finally included in this study, and their baseline demographics and laboratory parameters are shown in Table 1. The most common cause of end-stage renal disease was chronic glomerulonephritis. Mean eGFRs were 66.3, 65.1 , and $62.1 \mathrm{ml} / \mathrm{min} / 1.73 \mathrm{~m}^{2}$ at 1 month, 1 year, and 5 years after kidney transplantation, respectively. Approximately one-fourth $(53 / 214,24.8 \%)$ of the enrolled patients underwent ABO-incompatible kidney transplantation; they were older and more frequently received pretransplantation rituximab than those who underwent $\mathrm{ABO}$-compatible kidney transplantation (Supplemental Table 1). Acute rejection accounted for $63.0 \%(17 / 27)$ of the reasons for exclusions in our study; 13 and 4 cases were attributed to acute antibody-mediated rejection and acute T cell-mediated rejection, respectively (Supplemental Table 2).

\section{Phenotypic characterization of TLTs in transplanted kidneys}

PAS-stained graft tissues contained multiple TLT-like mononuclear cell infiltrates (Figure 2A), located in either subcapsular, perivascular, or periglomerular areas (Figure 2B-D), consistent with our previous study ${ }^{23}$. These infiltrates were composed of $\mathrm{T}$ and $\mathrm{B}$ cells, (Figure 2E-G), some of which were proliferating (Figure 2H), meeting our definition of TLTs. Among lymphocyte infiltrates detected in PAS-stained samples, $86.8 \%$ were confirmed as TLTs based on immunofluorescence (Figure 2I). In many TLTs, T and B cells were intermingled with one another (Figure 2E), but some TLTs harbored densely packed B cell clusters (Figure 2F and G). CD21-positive FDCs, i.e. stromal cells in charge of organizing B cell homeostasis in $\mathrm{TLTs}^{20}$, were also detected in some B cell clusters (Figure 2J and 2K). CXCL13 expression was also observed within TLTs and was colocalized with CD21 but not with CD45 (Figure 2L and 2M). Interestingly, T cell-dominant TLTs were 
Copyright 2021 by ASN, Published Ahead of Print on 11/1/21, Accepted/Unedited Version detected in the graft tissues of patients treated with pre-transplantation rituximab (Supplemental Figure 2B-D) in permissive areas for TLT formation described above. TLTs in these patients harbored far fewer B cells (Supplemental Figure 2B-D) than TLTs in aged kidneys and the kidney with chronic kidney disease (Supplemental Figure 2A) ${ }^{20}$, 23 , but yet had proliferating lymphocytes inside and therefore met the definition of TLTs.

\section{The prevalence and staging of TLTs in transplanted kidneys}

We next categorized TLT phenotypes utilizing the TLT staging strategy we recently established (see Methods for details). We observed stage I and stage II TLTs, but not stage III TLTs in protocol biopsies of transplanted kidneys (Figure 3). Notably, the prevalence of TLTs as well as their stages significantly changed after transplantation (Figure 4). In 0-hour biopsies, TLTs were found in only $3.8 \%$ of the samples. This prevalence increased to $46.9 \%$ at 1 month after kidney transplantation, and then slightly further within the first year $(53.4 \%$ and 58.4\% in 6- and 12-month biopsies, respectively). By contrast, the development of stage II TLTs was more gradual; their prevalence in 1-month biopsies was comparable to that of 0hour biopsies (1.4\% and 3.6\% in 0-hour and 1-month biopsies, respectively) and then began to increase steadily thereafter, reaching $8.6 \%$ in the 6-month biopsy samples (6.1-fold increase versus 0 -hour) and $18.9 \%$ in the 12 -month biopsy samples (13.5-fold increase versus 0-hour).

\section{Renal allograft outcomes in relation to the presence and stage of TLTs}

Next, we assessed renal allograft functions according to the presence and stages of TLTs at various time points of biopsies. The presence of TLTs, if stages were not taken into consideration, had no significant influence on late graft function (Supplemental Figure 3AF). However, when patients were divided according to TLT stage, those with stage II TLTs in 
Copyright 2021 by ASN, Published Ahead of Print on 11/1/21, Accepted/Unedited Version

the 6- or 12-month biopsies had significantly higher risk of death-censored renal function decline compared to those with no TLT (adjusted hazard ratios of 3.92 and 3.17 at the 6- and 12-month biopsies, $p=0.02$ and 0.015, respectively; Figure 5B, 5C and Table 2). eGFR over the 5 years after transplantation were also significantly lower in patients with stage II TLTs than in those with no TLT (adjusted mean differences of -14.35 and $-11.71 \mathrm{ml} / \mathrm{min} / 1.73$ $\mathrm{m}^{2}$ at the 6- and 12-month biopsies, $p=0.008$ and 0.012, respectively; Figure 5E, 5F and Table 3). In patients exhibiting stage II TLTs in the 1-month biopsy, the risk of late graft dysfunction was higher and mean eGFR at one-year post-transplantation was lower than in those without TLTs or in those with stage I TLTs, although without significant difference (Figure 5A, 5D and Table 2, 3). Sensitivity analyses of recipients who underwent ABOcompatible kidney transplantation consistently showed that the development of stage II TLT in the 6- or 12-month biopsy was associated with significantly higher risk of decline in graft function compared to those without TLTs (adjusted hazard ratios of 3.97 and 2.81 at the 6and 12-month biopsies, $p=0.030$ and 0.039, respectively; Supplemental Figure 4 and

\section{Supplemental Table 3, 4).}

Previous studies suggested a possible association between the presence of TLTs, especially B cell clusters, and the occurrence of allo-antibodies and subsequent antibody-mediated injury $^{32,33,51}$. In our cohort, donor specific antibodies at 1 year after transplantation were more frequently detected in patients with stage II TLTs than in those with no TLTs or stage I TLTs at the 12-month biopsies (Supplemental Figure 5). Nevertheless, no patient was diagnosed with biopsy-proven acute antibody-mediated rejection during 4 years of follow-up after the final protocol biopsy at 12 months. Seven patients had biopsy-proven acute T-cell mediated rejection during this period; however, these episodes were not associated with the prevalence of stage II TLTs at 12-month post-transplantation. 
Copyright 2021 by ASN, Published Ahead of Print on 11/1/21, Accepted/Unedited Version

\begin{abstract}
About one-third of our patients (66/214) experienced at least one episode of borderline T cellmediated rejection during the first year after kidney transplantation. Most of the patients were treated with steroid pulse therapy $(63 / 66,95.5 \%)$, and the trends of eGFR between patients with and without borderline rejection were not different (Supplemental Figure 6).
\end{abstract}

\title{
Risk factors for the development of stage II TLTs in the 12-month biopsies
}

Logistic regression analysis revealed that the use of pre-transplantation rituximab was the strong negative risk factor for the development of stage II TLTs in the 12-month biopsies (odds ratio of $0.17,95 \%$ confidence interval of $0.04-0.72, p=0.016$; Table 4). Pre-transplant rituximab administration suppressed stage II TLTs but did not affect the prevalence of stage I TLTs, regardless of biopsy time point (Figure 6 and Supplemental Table 5). The prevalence of stage II TLTs was lower in ABO-incompatible subgroup than in ABOcompatible subgroup, although without statistical significance (Supplemental Table 6). eGFR levels were maintained at similar levels in patients treated with pre-transplantation rituximab compared with those who were not, despite ABO incompatibility (Supplemental Figure 7).

The presence of donor specific antibody at 1 year after transplantation was positively associated with 12-month stage II TLTs (odds ratio of 7.63, 95\% confidence interval of 1.3642.91, $p=0.021$; Table 4). Borderline acute $\mathrm{T}$ cell-mediated rejection was not associated with the development of either stage I or II TLTs (Table 4 and Supplemental Table 5).

\section{The association between TLTs and Banff pathologic scores}

Finally, we investigated the relationship between TLT stages and Banff pathologic scores obtained at 12 months after kidney transplantation (Table 5). The presence of TLTs 
Copyright 2021 by ASN, Published Ahead of Print on 11/1/21, Accepted/Unedited Version

correlated with more pronounced interstitial inflammation at 12 months, presumably because TLTs themselves are regarded as interstitial inflammation according to the definition of Banff scores $^{42}$. Nevertheless, small stage II TLTs were occasionally found against the background of trivial Banff i scores (Supplemental Figure 8). Importantly, patients in the stage II TLT group exhibited significantly worse tubulitis, tubular atrophy, and interstitial fibrosis than did patients in the no TLT group ( $\mathrm{t}$ score of $0.12 \pm 0.36 \mathrm{vs} .0 .60 \pm 0.91$, ct score of $0.77 \pm 0.72 \mathrm{vs}$. $1.29 \pm 0.75$, and ci score of $0.70 \pm 0.63$ vs. 1.17 vs. $0.92 ; p<0.001,<0.001$, and 0.008 , respectively; Table 5). Patients with the stage I TLTs also showed worse tubulointerstitial inflammation, tubular atrophy, and interstitial fibrosis scores compared to those without TLTs (Table 5), although late graft function was similar between these groups (Figure 5F). Moreover, the presence of stage II TLTs at 12-month biopsies might be associated with future graft dysfunction, even in patients with quantitatively mild interstitial inflammation (adjusted hazard ratio of 2.60 and mean eGFR differences of $-11.2, p=0.048$ and 0.054 , respectively; Figure 7 and Table 6). Banff pathologic scores at 12-month biopsy were not significantly different between $\mathrm{ABO}$-compatible and $\mathrm{ABO}$-incompatible subgroups, except for $\mathrm{C} 4 \mathrm{~d}$, whose scores were clearly higher in recipients who underwent $\mathrm{ABO}$-incompatible transplantation (Supplemental Table 7). 
Copyright 2021 by ASN, Published Ahead of Print on 11/1/21, Accepted/Unedited Version

\section{Discussion}

In the present study, we investigated the prevalence and clinical relevance of TLTs in transplanted kidneys without the signs of rejection. We found that TLTs were frequent in rejection-free protocol biopsies and that their cellular and molecular phenotypes were similar to those found in aged patients ${ }^{20}$. By contrast to stage I TLTs that appeared as early as 1 month after kidney transplantation, stage II TLTs developed gradually over time and were independently associated with progressive graft dysfunction. These data suggest that advanced TLTs may help to stratify stable renal allografts without rejection into those with and without risk of functional deterioration.

The presence of lymphocyte clusters in the absence of rejection was first described in heart and lung allografts, in which protocol biopsies are performed more frequently; in these hearts and lungs, the prevalence of lymphocyte clusters ranged from 39 to $58 \%{ }^{52-57}$, similar to that of TLTs in our study (Figure 4). The clinical impacts of lymphocyte clusters on these allografts have been debated and controversial ${ }^{52-57}$. In the previous study, we showed that TLTs in the kidneys develop through at least three developmental stages irrespective of etiologies, and the developmental progression are associated with the severity of kidney injury in human utilizing surgically resected kidney samples ${ }^{23}$. In the present study, utilizing renal biopsy samples, we showed that the presence of stage II TLTs was associated with functional decline in graft function, while the presence of stage I TLTs was not. These results suggest that the presence of FDC, not of B cell infiltrations, is the determinant for future graft dysfunction, and may partly explain the inconsistent results of the clinical significance of graft-infiltrating B cells in transplanted kidneys ${ }^{24-29,32,34,58}$. We therefore propose evaluating TLTs using our staging strategy, especially focusing on the presence or absence of FDCs. Given that FDCs are found in heart allografts ${ }^{59,60}$, application of our TLT staging strategy 
Copyright 2021 by ASN, Published Ahead of Print on 11/1/21, Accepted/Unedited Version

may clarify hitherto unrevealed functional roles of lymphocyte clusters in other transplanted organs.

Time-dependent changes in the distribution of TLT stages provide valuable information regarding their evolution in renal allografts (Figure 4). Stage I TLTs rapidly developed within 1 month after kidney transplantation. By contrast, the prevalence of stage II TLTs did not change at this time point; rather, it increased in the 6- and 12-month biopsy samples. These findings are consistent with our rodent experimental data, where the proportion of advanced TLTs increased in a time-dependent manner after the injury ${ }^{23}$. Notably, we could not find histologic evidence of stage III TLTs, which had been documented in chronically rejected allograft explanted at more than 5 years after $\mathrm{KT}^{26,32,35}$. We speculate that 12 months were too short for ectopic germinal centers to be established and the intensity of graft inflammation in our cases was substantially lower than that in transplanted kidneys with chronic rejection.

Our TLT staging strategy distinguished between progressors and non-progressors, even among patients categorized as having Banff $\mathrm{i}$ scores of 0 or 1 . The paradox of kidney allograft with advanced TLTs being categorized as having minimal or mild interstitial inflammation is explained by differences in the grading systems used to score TLTs and interstitial inflammation in the Banff classification. Because Banff $i$ scores depend on the percentage of the area with inflammatory cell infiltration, biopsy samples with small, but FDC-containing TLTs would be classified as minimal or mild interstitial inflammation (Supplemental Figure 8). Another possible explanation is that interstitial infiltrates in subcapsular cortex and in areas of interstitial fibrosis were included in the assessment of TLTs, but not in the determination of Banff $\mathrm{i}$ score. Our data suggest that TLTs are 
Copyright 2021 by ASN, Published Ahead of Print on 11/1/21, Accepted/Unedited Version

inflammatory lesions that are qualitatively different from simple interstitial inflammation and therefore should be assessed in a different manner.

Although the underlying mechanisms were not investigated here, analysis of Banff pathologic scores identified sustained tubular injury as a possible cause of progressive graft dysfunction in patients with stage II TLTs (Table 5). Consistent with this hypothesis, a rodent kidney transplantation model study demonstrated that B cells in TLTs promoted tubulointerstitial fibrosis, possibly by secreting fibrosis-related cytokines ${ }^{61}$. Other studies demonstrated that TLTs were associated with the formation of alloantibodies ${ }^{26,62}$, and the intensity of antibodymediated alloimmune responses correlated with the maturation status of $\operatorname{TLTs}^{32,} 33$, suggesting a link between advanced TLTs and antibody-mediated graft injury. In the present study, however, no patient showed biopsy-proven antibody-mediated rejection, although donor-specific antibodies were more frequently observed in patients with stage II TLTs (Table 4 and Supplemental Figure 5). Moreover, stage II TLTs were not associated with any pathologic features suggestive of antibody-mediated injury such as glomerulitis (g score), peritubular capillaritis (ptc score), or C4d staining at 12 months post-transplantation (Table 5), consistent with findings of a previous report ${ }^{63}$. Taken together, these data suggest that stage II TLTs contributed to graft dysfunction presumably via tubular inflammation and fibrosis, at least in the first year after kidney transplantation. The association between advanced TLTs and antibody-mediated rejection should be clarified in further investigations.

The effects of rituximab on the development of TLTs have rarely been investigated. In the present study, the administration of pre-transplantation rituximab dramatically reduced stage II TLTs up to a year after kidney transplantation (Figure 6). These findings are mechanistically reasonable, given the capacity of rituximab to deplete circulating B cells for 
12 months or longer ${ }^{64}$. Interestingly, a retrospective study showed that post-transplantation rituximab did not result in the clearance of intra-graft TLTs $^{58}$. Similar findings were consistently reported in other conditions such as autoimmune diseases ${ }^{65-71}$, suggesting the importance of the timing of rituximab infusion for controlling TLT formation. Furthermore, patients treated with pre-transplantation rituximab maintained similar graft function over 5 years after kidney transplantation, comparable to those who did not, even though the immunologic risk was higher in the ABO-incompatible, rituximab-treated group (Supplemental Figure 7). Nevertheless, it remains uncertain whether this effect was due to the reduction of stage II TLTs or other unrevealed mechanisms of rituximab. It is also possible that plasma exchange and/or intravenous immunoglobulin, administered along with rituximab, may be associated with suppression of advanced TLTs.

It is noteworthy that most patients treated with pre-transplantation rituximab were ABOincompatible subgroups $(50 / 57,87.7 \%)$, indicating that the significant differences in the prevalence of stage II TLTs between ABO-compatible and ABO-incompatible subgroups might be due to the different baseline demographics and immunologic risks rather than the use of pre-transplantation rituximab. It was difficult to investigate the effects of pretransplantation rituximab on stage II TLTs among recipients who underwent ABOcompatible transplantation, because of the small number of rituximab-treated recipients in this population $(7 / 161,4.3 \%)$. Given that pre-transplantation rituximab is prescribed exclusively for patients with high immunologic risks, distinguishing the effects of rituximab and immunologic profiles on stage II TLTs would be extremely difficult in real world.

Older age, an important risk factor for developing TLTs ${ }^{20}$, was not associated with their formation in the present study (Table 4). One of the reasons for this discrepancy could be the 
Copyright 2021 by ASN, Published Ahead of Print on 11/1/21, Accepted/Unedited Version

fact that both kidney donor and recipients were much younger compared with those recruited in our previous $\operatorname{study}^{20}$ (i.e. a mean age of 58 (donor) and 48 (recipients) vs. 70 years (previous study)). We speculate that sustained inflammatory stimuli from immunologic differences, rather than the age of patients, appear to be a more powerful inducer of TLTs in the setting of kidney transplantation.

A limitation of this study should be mentioned. Approximately 12\% (108/856) of biopsy samples were missing in this study, mostly because of refusals by recipients $(4[1.9 \%], 22$ [10.3\%], 51 [23.8\%], and 31 [14.5\%] missed samples at 0-hour, 1-, 6-, and 12-month posttransplantation, respectively); these missingness raises a possibility of unexpected selection bias. Nonetheless, we speculate that the proportion of missing cases were relatively small, considering the difficulties in obtaining serial protocol biopsies from recipients maintaining stable graft function. Furthermore, the baseline characteristics and clinical parameters were mostly comparable between recipients who underwent protocol biopsy and those who did not (data not shown). Therefore, the impacts of the missing cases on the overall results might be trivial.

In conclusion, we demonstrated that the intra-graft detection of stage II TLTs was independently associated with progressive decline in renal allograft function. Prospective studies are needed to confirm whether our novel TLT staging strategy has the potential to serve not only as a valuable tool for systematic classification, but also as a predictor of transplant functional decline. Further investigations are also needed to determine whether therapeutic strategies to prevent the development and maturation of TLTs could lead to better long-term graft outcomes in kidney transplant recipients. 
Copyright 2021 by ASN, Published Ahead of Print on 11/1/21, Accepted/Unedited Version

\section{Author contributions}

Y.H.L., Y.S. and M.Y. designed the study; Y.H.L. and Y.S. carried out experiments; Y.H.L., Y.S., M.S., M.S., S.Y., A.K., N.F., S.S., T.H. and M.Y. collected and analyzed the data; Y.H.L. and S.F. performed statistical analyses; Y.H.L., Y.S., S.H.L., B.P., F.J. and M.Y. drafted and revised the paper; all authors approved the final version of the manuscript.

\section{Acknowledgements}

We thank Ms. Kasumoto, Ms. Tomita, Ms. Sakurai, Ms. Nakayama, and Ms. Ozone for their excellent technical assistance.

\section{Funding}

This research was supported by the Japan Agency for Medical Research and Development (AMED) under Grant Number 20gm1210009, JP20gm5010002 and JP120gm0610011; partially by grants from the TMK Project, KAKENHI Grant-in-Aids for Scientific Research B (26293202, 17H04187), Grant in Aid for Scientific Research on Innovative Areas "Stem Cell Aging and Disease (17H05642)" and "Lipoquality (18H04673)", Grant-in-Aid for Young Scientists (B) from the Japan Society for the Promotion of Science (JSPS), the Translational Research program, Strategic Promotion for practical application of INnovative medical Technology (TR-SPRINT) from AMED; grants from the Uehara Memorial Foundation, Takeda Science Foundation, and the Sumitomo Foundation. This work was also partly supported by World Premier International Research Center Initiative (WPI), MEXT, Japan. This study was co-financed by the German Research Foundation (DFG: SFB/TRR57 and SFB/TRR219, BO3755/3-1 and BO3755/6-1), the German Ministry of Education and Research (BMBF: STOP-FSGS-01GM1518A), the 
Copyright 2021 by ASN, Published Ahead of Print on 11/1/21, Accepted/Unedited Version

RWTH Interdisciplinary Centre for Clinical Research (IZKF: O3-7), and the National Research Foundation of Korea (NRF-2021R1G1A1014115).

\section{Disclosure}

YS is employed by TMK project. MY receives research grants from Astellas, Chugai, Daiichi Sankyo, Fujiyakuhin, Kyowa Hakko Kirin, Mitsubishi Tanabe, MSD, Nippon Boehringer Ingelheim, and Torii; Honoraria from Astellas, Kyowa Kirin, Chugai, and others for lecture honoraria; and Other Interests/Relationships with Japanese Society of Nephrology, and International Society of Nephrology. S. Fukuma reports Consultancy Agreements with Kyowa Hakko Kirin, Rege Nephro; Research Funding from Kyowa Hakko Kirin, CANCER SCAN; Honoraria from Kyowa Hakko Kirin; and Scientific Advisor or Membership with Kyowa Hakko Kirin. S. Yamamoto reports Other Interests/Relationships with the Japanese society of Nephrology, and The Japanese society for Dialysis Therapy. T. Habuchi reports Research Funding from Kyowa Kirin Co., Astellas Pharma Inc., Bayer AG., Chugai Pharmaceutical Co.; and Honoraria from Kyowa Kirin Co., Astellas Pharma Inc., Bayer AG., Chugai Pharmaceutical Co. J. Floege reports Consultancy Agreements with Calliditas, Travere, Novartis, Idorsia, Omeros, Bayer, AstraZeneca; and Honoraria from Calliditas, Travere, Novartis, Idorsia, Omeros, Bayer, AstraZeneca, Vifor, and Fresenius. All remaining authors report no conflicts of interest. 
Copyright 2021 by ASN, Published Ahead of Print on 11/1/21, Accepted/Unedited Version

\section{References}

1. Lamb KE, Lodhi S, Meier-Kriesche HU: Long-term renal allograft survival in the United States: a critical reappraisal. Am J Transplant 11: 450-462, 2011

2. Coemans M, Susal C, Dohler B, Anglicheau D, Giral M, Bestard O, et al.: Analyses of the short- and long-term graft survival after kidney transplantation in Europe between 1986 and 2015. Kidney Int 94: 964-973, 2018

3. Dahle DO, Mjoen G, Oqvist B, Scharnagl H, Weihrauch G, Grammer T, et al.: Inflammation-associated graft loss in renal transplant recipients. Nephrol Dial Transplant 26: 3756-3761, 2011

4. Galichon P, Xu-Dubois YC, Finianos S, Hertig A, Rondeau E: Clinical and histological predictors of long-term kidney graft survival. Nephrol Dial Transplant 28: 13621370,2013

5. Solhjou Z, Athar H, Xu Q, Abdi R: Emerging therapies targeting intra-organ inflammation in transplantation. Am J Transplant 15: 305-311, 2015

6. Batal I, De Serres SA, Safa K, Bijol V, Ueno T, Onozato ML, et al.: Dendritic Cells in Kidney Transplant Biopsy Samples Are Associated with T Cell Infiltration and Poor Allograft Survival. J Am Soc Nephrol 26: 3102-3113, 2015

7. Shishido S, Asanuma H, Nakai H, Mori Y, Satoh H, Kamimaki I, et al.: The impact of repeated subclinical acute rejection on the progression of chronic allograft nephropathy. J Am Soc Nephrol 14: 1046-1052, 2003

8. Nankivell BJ, Borrows RJ, Fung CL, O'Connell PJ, Allen RD, Chapman JR: Natural history, risk factors, and impact of subclinical rejection in kidney transplantation. Transplantation 78: 242-249, 2004

9. Moreso F, Ibernon M, Goma M, Carrera M, Fulladosa X, Hueso M, et al.: Subclinical rejection associated with chronic allograft nephropathy in protocol biopsies as a risk 
Copyright 2021 by ASN, Published Ahead of Print on 11/1/21, Accepted/Unedited Version factor for late graft loss. Am J Transplant 6: 747-752, 2006

10. Heilman RL, Devarapalli Y, Chakkera HA, Mekeel KL, Moss AA, Mulligan DC, et al.: Impact of subclinical inflammation on the development of interstitial fibrosis and tubular atrophy in kidney transplant recipients. Am J Transplant 10: 563-570, 2010

11. Park WD, Griffin MD, Cornell LD, Cosio FG, Stegall MD: Fibrosis with inflammation at one year predicts transplant functional decline. J Am Soc Nephrol 21: 1987-1997, 2010

12. Neyt K, Perros F, GeurtsvanKessel CH, Hammad H, Lambrecht BN: Tertiary lymphoid organs in infection and autoimmunity. Trends Immunol 33: 297-305, 2012

13. Pitzalis C, Jones GW, Bombardieri M, Jones SA: Ectopic lymphoid-like structures in infection, cancer and autoimmunity. Nat Rev Immunol 14: 447-462, 2014

14. Koenig A, Thaunat O: Lymphoid Neogenesis and Tertiary Lymphoid Organs in Transplanted Organs. Front Immunol 7: 646, 2016

15. Sato Y, Yanagita M: Immunology of the ageing kidney. Nat Rev Nephrol 15: 625-640, 2019

16. Ansel KM, Ngo VN, Hyman PL, Luther SA, Forster R, Sedgwick JD, et al.: A chemokine-driven positive feedback loop organizes lymphoid follicles. Nature 406: $309-314,2000$

17. Luther SA, Lopez T, Bai W, Hanahan D, Cyster JG: BLC expression in pancreatic islets causes B cell recruitment and lymphotoxin-dependent lymphoid neogenesis. Immunity $12: 471-481,2000$

18. Buckley CD, Barone F, Nayar S, Benezech C, Caamano J: Stromal cells in chronic inflammation and tertiary lymphoid organ formation. Annu Rev Immunol 33: 715-745, 2015

19. Barone F, Gardner DH, Nayar S, Steinthal N, Buckley CD, Luther SA: Stromal 
Copyright 2021 by ASN, Published Ahead of Print on 11/1/21, Accepted/Unedited Version

Fibroblasts in Tertiary Lymphoid Structures: A Novel Target in Chronic Inflammation. Front Immunol 7: 477, 2016

20. Sato Y, Mii A, Hamazaki Y, Fujita H, Nakata H, Masuda K, et al.: Heterogeneous fibroblasts underlie age-dependent tertiary lymphoid tissues in the kidney. JCI Insight 1: e87680, 2016

21. Sato Y, Yanagita M: Resident fibroblasts in the kidney: a major driver of fibrosis and inflammation. Inflamm Regen 37: 17, 2017

22. Sato Y, Yanagita M: Functional heterogeneity of resident fibroblasts in the kidney. Proc Jpn Acad Ser B Phys Biol Sci 95: 468-478, 2019

23. Sato Y, Boor P, Fukuma S, Klinkhammer BM, Haga H, Ogawa O, et al.: Developmental stages of tertiary lymphoid tissue reflect local injury and inflammation in mouse and human kidneys. Kidney Int 98: 448-463, 2020

24. Sarwal M, Chua MS, Kambham N, Hsieh SC, Satterwhite T, Masek M, et al.: Molecular heterogeneity in acute renal allograft rejection identified by DNA microarray profiling. N Engl J Med 349: 125-138, 2003

25. Hippen BE, DeMattos A, Cook WJ, Kew CE, 2nd, Gaston RS: Association of CD20+ infiltrates with poorer clinical outcomes in acute cellular rejection of renal allografts. Am J Transplant 5: 2248-2252, 2005

26. Thaunat O, Field AC, Dai J, Louedec L, Patey N, Bloch MF, et al.: Lymphoid neogenesis in chronic rejection: evidence for a local humoral alloimmune response. Proc Natl Acad Sci U S A 102: 14723-14728, 2005

27. Bagnasco SM, Tsai W, Rahman MH, Kraus ES, Barisoni L, Vega R, et al.: CD20positive infiltrates in renal allograft biopsies with acute cellular rejection are not associated with worse graft survival. Am J Transplant 7: 1968-1973, 2007

28. Kayler LK, Lakkis FG, Morgan C, Basu A, Blisard D, Tan HP, et al.: Acute cellular 
Copyright 2021 by ASN, Published Ahead of Print on 11/1/21, Accepted/Unedited Version rejection with CD20-positive lymphoid clusters in kidney transplant patients following lymphocyte depletion. Am J Transplant 7: 949-954, 2007

29. Scheepstra C, Bemelman FJ, van der Loos C, Rowshani AT, van Donselaar-Van der Pant KA, Idu MM, et al.: B cells in cluster or in a scattered pattern do not correlate with clinical outcome of renal allograft rejection. Transplantation 86: 772-778, 2008

30. Zarkhin V, Kambham N, Li L, Kwok S, Hsieh SC, Salvatierra O, et al.: Characterization of intra-graft B cells during renal allograft rejection. Kidney Int 74: 664-673, 2008

31. Hwang HS, Song JH, Hyoung BJ, Lee SY, Jeon YJ, Kang SH, et al.: Clinical impacts of $\mathrm{CD} 38+\mathrm{B}$ cells on acute cellular rejection with $\mathrm{CD} 20+\mathrm{B}$ cells in renal allograft. Transplantation 89: 1489-1495, 2010

32. Thaunat O, Patey N, Caligiuri G, Gautreau C, Mamani-Matsuda M, Mekki Y, et al.: Chronic rejection triggers the development of an aggressive intragraft immune response through recapitulation of lymphoid organogenesis. J Immunol 185: 717-728, 2010

33. Cheng J, Torkamani A, Grover RK, Jones TM, Ruiz DI, Schork NJ, et al.: Ectopic B-cell clusters that infiltrate transplanted human kidneys are clonal. Proc Natl Acad Sci US A 108: 5560-5565, 2011

34. Abbas K, Mubarak M, Zafar MN, Aziz T, Abbas H, Muzaffar R, et al.: Plasma cell-rich acute rejections in living-related kidney transplantation: a clinicopathological study of 50 cases. Clin Transplant 29: 835-841, 2015

35. Xu X, Han Y, Wang Q, Cai M, Qian Y, Wang X, et al.: Characterisation of Tertiary Lymphoid Organs in Explanted Rejected Donor Kidneys. Immunol Invest 45: 38-51, 2016

36. Segerer S, Schlondorff D: B cells and tertiary lymphoid organs in renal inflammation. Kidney Int 73: 533-537, 2008 
Copyright 2021 by ASN, Published Ahead of Print on 11/1/21, Accepted/Unedited Version

37. Thaunat O: Pathophysiologic significance of B-cell clusters in chronically rejected grafts. Transplantation 92: 121-126, 2011

38. Hsiao HM, Li W, Gelman AE, Krupnick AS, Kreisel D: The Role of Lymphoid Neogenesis in Allografts. Am J Transplant 16: 1079-1085, 2016

39. Nasr IW, Reel M, Oberbarnscheidt MH, Mounzer RH, Baddoura FK, Ruddle NH, et al.: Tertiary lymphoid tissues generate effector and memory $\mathrm{T}$ cells that lead to allograft rejection. Am J Transplant 7: 1071-1079, 2007

40. Brown K, Sacks SH, Wong W: Tertiary lymphoid organs in renal allografts can be associated with donor-specific tolerance rather than rejection. Eur J Immunol 41: 8996,2011

41. Miyajima M, Chase CM, Alessandrini A, Farkash EA, Della Pelle P, Benichou G, et al.: Early acceptance of renal allografts in mice is dependent on foxp3(+) cells. $A m J$ Pathol 178: 1635-1645, 2011

42. Haas M, Loupy A, Lefaucheur C, Roufosse C, Glotz D, Seron D, et al.: The Banff 2017 Kidney Meeting Report: Revised diagnostic criteria for chronic active T cell-mediated rejection, antibody-mediated rejection, and prospects for integrative endpoints for next-generation clinical trials. Am J Transplant 18: 293-307, 2018

43. Jeong HJ: Diagnosis of renal transplant rejection: Banff classification and beyond. Kidney Res Clin Pract 39: 17-31, 2020

44. Loupy A, Vernerey D, Tinel C, Aubert O, Duong van Huyen JP, Rabant M, et al.: Subclinical Rejection Phenotypes at 1 Year Post-Transplant and Outcome of Kidney Allografts. J Am Soc Nephrol 26: 1721-1731, 2015

45. Fujiyama N, Satoh S, Saito M, Numakura K, Inoue T, Yamamoto R, et al.: Impact of persistent preformed and de novo donor-specific antibodies detected at 1 year after kidney transplantation on long-term graft survival in Japan: a retrospective study. Clin 
Copyright 2021 by ASN, Published Ahead of Print on 11/1/21, Accepted/Unedited Version Exp Nephrol 23: 1398-1406, 2019

46. Horio M, Imai E, Yasuda $\mathrm{Y}$, Watanabe $\mathrm{T}$, Matsuo $\mathrm{S}$ : Modification of the CKD epidemiology collaboration (CKD-EPI) equation for Japanese: accuracy and use for population estimates. Am J Kidney Dis 56: 32-38, 2010

47. Komine N, Satoh S, Saito M, Numakura K, Inoue T, Tsuruta H, et al.: Influence of CYP3A5 genetic differences in tacrolimus on quantitative interstitial fibrosis and long-term graft function in kidney transplant recipients. Int Immunopharmacol 58: $57-63,2018$

48. Sato Y, Tamura M, Yanagita M: Tertiary lymphoid tissues: a regional hub for kidney inflammation. Nephrol Dial Transplant 37: 1-8, 2021

49. Iguchi T, Takaori K, Mii A, Sato Y, Suzuki Y, Yoshifuji H, et al.: Glucocorticoid receptor expression in resident and hematopoietic cells in IgG4-related disease. Mod Pathol 31: 890-899, 2018

50. Graubard BI, Korn EL: Modelling the sampling design in the analysis of health surveys. Stat Methods Med Res 5: 263-281, 1996

51. Thaunat O, Graff-Dubois S, Brouard S, Gautreau C, Varthaman A, Fabien N, et al.: Immune responses elicited in tertiary lymphoid tissues display distinctive features. PLoS One 5: e11398, 2010

52. Hasegawa T, Iacono A, Yousem SA: The significance of bronchus-associated lymphoid tissue in human lung transplantation: is there an association with acute and chronic rejection? Transplantation 67: 381-385, 1999

53. Yamani MH, Ratliff NB, Starling RC, Tuzcu EM, Yu Y, Cook DJ, et al.: Quilty lesions are associated with increased expression of vitronectin receptor (alphavbeta3) and subsequent development of coronary vasculopathy. J Heart Lung Transplant 22: 687690,2003 
Copyright 2021 by ASN, Published Ahead of Print on 11/1/21, Accepted/Unedited Version

54. Chu KE, Ho EK, de la Torre L, Vasilescu ER, Marboe CC: The relationship of nodular endocardial infiltrates (Quilty lesions) to survival, patient age, anti-HLA antibodies, and coronary artery disease following heart transplantation. Cardiovasc Pathol 14: 219-224, 2005

55. Hiemann NE, Knosalla C, Wellnhofer E, Lehmkuhl HB, Hetzer R, Meyer R: Quilty indicates increased risk for microvasculopathy and poor survival after heart transplantation. J Heart Lung Transplant 27: 289-296, 2008

56. Hiemann NE, Knosalla C, Wellnhofer E, Lehmkuhl HB, Hetzer R, Meyer R: Quilty in biopsy is associated with poor prognosis after heart transplantation. Transpl Immunol 19: 209-214, 2008

57. Zakliczynski M, Nozynski J, Konecka-Mrowka D, Pyka L, Trybunia D, Swierad M, et al.: Quilty effect correlates with biopsy-proven acute cellular rejection but does not predict transplanted heart coronary artery vasculopathy. J Heart Lung Transplant 28: $255-259,2009$

58. Thaunat O, Patey N, Gautreau C, Lechaton S, Fremeaux-Bacchi V, Dieu-Nosjean MC, et al.: B cell survival in intragraft tertiary lymphoid organs after rituximab therapy. Transplantation 85: 1648-1653, 2008

59. Sattar HA, Husain AN, Kim AY, Krausz T: The presence of a CD21+ follicular dendritic cell network distinguishes invasive Quilty lesions from cardiac acute cellular rejection. Am J Surg Pathol 30: 1008-1013, 2006

60. Di Carlo E, D'Antuono T, Contento S, Di Nicola M, Ballone E, Sorrentino C: Quilty effect has the features of lymphoid neogenesis and shares CXCL13-CXCR5 pathway with recurrent acute cardiac rejections. Am J Transplant 7: 201-210, 2007

61. Tse GH, Johnston CJ, Kluth D, Gray M, Gray D, Hughes J, et al.: Intrarenal B Cell Cytokines Promote Transplant Fibrosis and Tubular Atrophy. Am J Transplant 15: 
Copyright 2021 by ASN, Published Ahead of Print on 11/1/21, Accepted/Unedited Version 3067-3080, 2015

62. Thaunat O, Graff-Dubois S, Fabien N, Duthey A, Attuil-Audenis V, Nicoletti A, et al.: A stepwise breakdown of B-cell tolerance occurs within renal allografts during chronic rejection. Kidney Int 81: 207-219, 2012

63. Cippa PE, Liu J, Sun B, Kumar S, Naesens M, McMahon AP: A late B lymphocyte action in dysfunctional tissue repair following kidney injury and transplantation. Nat Commun 10: 1157, 2019

64. Barnett AN, Hadjianastassiou VG, Mamode N: Rituximab in renal transplantation. Transpl Int 26: 563-575, 2013

65. Vos K, Thurlings RM, Wijbrandts CA, van Schaardenburg D, Gerlag DM, Tak PP: Early effects of rituximab on the synovial cell infiltrate in patients with rheumatoid arthritis. Arthritis Rheum 56: 772-778, 2007

66. Quartuccio L, Fabris M, Moretti M, Barone F, Bombardieri M, Rupolo M, et al.: Resistance to rituximab therapy and local BAFF overexpression in Sjogren's syndrome-related myoepithelial sialadenitis and low-grade parotid B-cell lymphoma. Open Rheumatol J 2: 38-43, 2008

67. Rosengren S, Wei N, Kalunian KC, Zvaifler NJ, Kavanaugh A, Boyle DL: Elevated autoantibody content in rheumatoid arthritis synovia with lymphoid aggregates and the effect of rituximab. Arthritis Res Ther 10: R105, 2008

68. Thurlings RM, Vos K, Wijbrandts CA, Zwinderman AH, Gerlag DM, Tak PP: Synovial tissue response to rituximab: mechanism of action and identification of biomarkers of response. Ann Rheum Dis 67: 917-925, 2008

69. Pijpe J, Meijer JM, Bootsma H, van der Wal JE, Spijkervet FK, Kallenberg CG, et al.: Clinical and histologic evidence of salivary gland restoration supports the efficacy of rituximab treatment in Sjogren's syndrome. Arthritis Rheum 60:3251-3256, 2009 
Copyright 2021 by ASN, Published Ahead of Print on 11/1/21, Accepted/Unedited Version

70. Hamza N, Bootsma H, Yuvaraj S, Spijkervet FK, Haacke EA, Pollard RP, et al.: Persistence of immunoglobulin-producing cells in parotid salivary glands of patients with primary Sjogren's syndrome after B cell depletion therapy. Ann Rheum Dis 71: $1881-1887,2012$

71. Regard L, Martin C, Zemoura L, Geolier V, Sage E, Burgel PR: Peribronchial tertiary lymphoid structures persist after rituximab therapy in patients with cystic fibrosis. $J$ Clin Pathol 71: 752-753, 2018 
Copyright 2021 by ASN, Published Ahead of Print on 11/1/21, Accepted/Unedited Version

Table 1. Baseline characteristics and clinical parameters of enrolled patients

Number of patients

Age (year)

Gender (Male, \%)

Body mass index $\left(\mathrm{kg} / \mathrm{m}^{2}\right)$

Etiology of end-stage renal disease (n, \%)

Chronic glomerulonephritis

Diabetes mellitus

Hypertension

Polycystic kidney disease

Others $^{\text {a }}$

Time on dialysis (month)

Preemptive kidney transplantation (n, \%)

Number of HLA mismatching (n)

Positive crossmatch

ABO-incompatible kidney transplantation (n, \%)

Pre-transplantation rituximab (n, \%)

Cold ischemic time (minute)

Warm ischemic time (minute)

Induction immunosuppressant (n, \%)

Basiliximab

Maintenance immunosuppressant (n, \%) ${ }^{b}$

Prednisolone

Tacrolimus

Mycophenolate mofetil

Borderline T cell-mediated rejection $(\mathrm{n}, \%)^{\mathrm{c}}$

Donor specific antibody at 1 -year post-transplantation (n, \% $)^{d}$

Class I

\section{4}

$48.8 \pm 12.5$

$139(65.0)$

$22.4 \pm 3.7$

$122(57.0)$

$36(16.8)$

$16(7.5)$

$14(6.5)$

$26(12.2)$

$18.0[5.0,48.8]$

$40(18.7)$

$3.3 \pm 1.5$

$14(6.5)$

$53(24.8)$

$57(26.6)$

$144.0 \pm 37.6$

$5.0 \pm 3.7$

$214(100)$

$171(79.9)$

$214(100)$

$214(100)$

$66(30.8)$

$13 / 207(6.3)$

$7(53.8)$ 
Copyright 2021 by ASN, Published Ahead of Print on 11/1/21, Accepted/Unedited Version

\begin{tabular}{lc}
\hline Class II & $7(53.8)$ \\
Post-transplant eGFR $\left(\mathrm{ml} / \mathrm{min} / 1.73 \mathrm{~m}^{2}\right)$ & \\
1-month & $66.3 \pm 20.6$ \\
1-year & $65.1 \pm 18.9$ \\
$\quad$-year & $62.1 \pm 21.6$ \\
Donor age (year) & $58.3 \pm 9.9$ \\
Donor gender (Male, \%) & $82(38.3)$ \\
Pre-transplantation donor eGFR $\left(\mathrm{ml} / \mathrm{min} / 1.73 \mathrm{~m}^{2}\right)$ & $103.0 \pm 10.8$
\end{tabular}

Data are expressed as mean \pm standard deviation or the number of patients (percentage). Time on dialysis is non-normally distributed and is expressed as median $\left[1^{\text {st }}\right.$ and $3^{\text {rd }}$ interquartile range].

${ }^{a}$ Others include chronic tubulointerstitial nephritis, gestational hypertension, vesicoureteral reflux disease, sepsis, cystinuria, and bone marrow transplant nephropathy.

${ }^{\mathrm{b}}$ Data obtained at the time of outpatient visit 1 year after transplantation.

${ }^{c}$ At least one episode during the first year after transplantation.

${ }^{\mathrm{d}}$ Not assessed in 7 recipients.

Abbreviations: HLA, human leukocyte antigen; eGFR, estimated glomerular filtration rate. 
Copyright 2021 by ASN, Published Ahead of Print on 11/1/21, Accepted/Unedited Version

Table 2. Hazard ratios of the stages of tertiary lymphoid tissues for death-censored renal function decline

\begin{tabular}{lcccc}
\hline & TLT stages & No. of events $^{\text {a }}(\%)$ & Adjusted HR $^{\text {b }}(95 \%$ CI $)$ & $p$ value \\
\hline \multirow{3}{*}{ 1-month } & No TLT $(\mathrm{n}=102)$ & $19(18.6)$ & Reference & - \\
& Stage I $(\mathrm{n}=83)$ & $18(21.7)$ & $1.44(0.72$ to 2.87$)$ & 0.309 \\
& Stage II $(\mathrm{n}=7)$ & $3(42.9)$ & $3.60(0.96$ to 13.50$)$ & 0.058 \\
\hline \multirow{3}{*}{ 6-month } & No TLT $(\mathrm{n}=76)$ & $10(13.2)$ & Reference & - \\
& Stage I $(\mathrm{n}=73)$ & $15(20.5)$ & $1.49(0.63$ to 3.53$)$ & 0.370 \\
& Stage II $(\mathrm{n}=14)$ & $6(42.9)$ & $3.92(1.23$ to 12.47$)$ & 0.020 \\
\hline \multirow{3}{*}{ 12-month } & No TLT $(\mathrm{n}=77)$ & $11(14.3)$ & Reference & - \\
& Stage I $(\mathrm{n}=73)$ & $13(17.8)$ & $1.05(0.44$ to 2.51$)$ & 0.914 \\
& Stage II $(\mathrm{n}=35)$ & $14(40.0)$ & $3.17(1.25$ to 8.02$)$ & 0.015 \\
\hline
\end{tabular}

a Renal function decline was defined as a decline of at least 30\% in the eGFR from 1-year post-transplant graft function.

b The comparisons between groups are performed by Cox regression analysis with multiple adjustments for confounders including age, sex, the presence of diabetes after transplantation, ABO incompatibility, positive crossmatch, the presence of donor specific antibody at 1-year post-transplantation, and pre-transplantation donor eGFR.

Abbreviations: TLT, tertiary lymphoid tissue; HR, hazard ratio; CI, confidence interval; eGFR, estimated glomerular filtration rate. 
Copyright 2021 by ASN, Published Ahead of Print on 11/1/21, Accepted/Unedited Version

Table 3. Association between the stages of tertiary lymphoid tissues and graft function

\begin{tabular}{cccc}
\hline Biopsy time point & TLT stages & $\begin{array}{c}\text { Adjusted difference in eGFR a } \\
(95 \% \text { CI })\end{array}$ & $p$ value \\
\hline \multirow{2}{*}{ 1-month } & No TLT $(\mathrm{n}=102)$ & Reference & - \\
& Stage I $(\mathrm{n}=83)$ & $-0.24(-6.04$ to 5.56$)$ & 0.935 \\
& Stage II $(\mathrm{n}=7)$ & $-13.16(-32.60$ to 6.29$)$ & 0.185 \\
\hline \multirow{2}{*}{ 6-month } & No TLT $(\mathrm{n}=76)$ & Reference & - \\
& Stage I $(\mathrm{n}=73)$ & $-4.07(-10.07$ to 1.93$)$ & 0.183 \\
& Stage II $(\mathrm{n}=14)$ & $-14.35(-24.93$ to -3.76$)$ & 0.008 \\
\hline \multirow{2}{*}{ 12-month } & No TLT $(\mathrm{n}=77)$ & Reference & - \\
& Stage I $(\mathrm{n}=73)$ & $-1.16(-5.78$ to 3.47$)$ & 0.624 \\
& Stage II $(\mathrm{n}=35)$ & $-11.71(-20.84$ to -2.58$)$ & 0.012
\end{tabular}

$\overline{\mathrm{a}}$ The comparisons between groups are performed by linear mixed effect models with multiple adjustments for confounders including recipient age and gender, donor age and gender, recipients body mass index, preemptive kidney transplantation, the presence of diabetes after transplantation, the number of HLA mismatching, positive crossmatch, the use of immunosuppressant, baseline graft function, the presence of donor specific antibody at 1-year post-transplantation, and pre-transplantation donor eGFR. Baseline graft function was set as eGFR levels for each time point. The differences in eGFR are calculated by comparing eGFR at baseline and eGFR at 4-5 years after kidney transplantation.

Abbreviations: TLT, tertiary lymphoid tissue; eGFR, estimated glomerular filtration rate; CI, confidence interval; HLA, human leukocyte antigen. 
Copyright 2021 by ASN, Published Ahead of Print on 11/1/21, Accepted/Unedited Version

Table 4. Multivariable analyses of risk factors for the development of stage II tertiary lymphoid tissues in 12-month protocol biopsies

\begin{tabular}{|c|c|c|}
\hline Variables & OR $(95 \% \mathrm{CI})$ & $p$ value \\
\hline Recipient age (per 10-year increase) & $1.08(0.74-1.59)$ & 0.696 \\
\hline Donor age (per 10-year increase) & $0.69(0.38-1.24)$ & 0.210 \\
\hline Recipient gender (male) & $0.93(0.30-2.85)$ & 0.892 \\
\hline Donor gender (male) & $1.84(0.63-5.32)$ & 0.263 \\
\hline Body mass index & $0.97(0.85-1.11)$ & 0.679 \\
\hline Diabetes mellitus & $1.70(0.63-4.55)$ & 0.292 \\
\hline Number of HLA mismatching (per one mismatch increase) & $0.90(0.65-1.24)$ & 0.519 \\
\hline Positive crossmatch & $0.86(0.11-7.06)$ & 0.891 \\
\hline Pre-transplantation rituximab & $0.17(0.04-0.72)$ & 0.016 \\
\hline Steroid maintenance therapy at 1-year post-transplantation & $0.55(0.20-1.57)$ & 0.265 \\
\hline Cold ischemic time (per ten-minute increase) & $1.02(0.95-1.10)$ & 0.568 \\
\hline Warm ischemic time (per an minute increase) & $0.91(0.76-1.09)$ & 0.299 \\
\hline Donor specific antibody at 1 -year post-transplantation & $7.63(1.36-42.91)$ & 0.021 \\
\hline 12-month eGFR (per $10 \mathrm{ml} / \mathrm{min} / 1.73 \mathrm{~m}^{2}$ increase) & $0.96(0.71-1.23)$ & 0.779 \\
\hline Donor eGFR (per $10 \mathrm{ml} / \mathrm{min} / 1.73 \mathrm{~m}^{2}$ increase) & $0.61(0.35-1.06)$ & 0.080 \\
\hline Borderline $\mathrm{T}$ cell-mediated rejection & $1.88(0.73-4.85)$ & 0.192 \\
\hline
\end{tabular}

ABO incompatibility was not used as variables because of its significant correlation with the use of pre-transplantation rituximab.

Abbreviations: OR, odds ratio; CI, confidence interval; HLA, human leukocyte antigen; eGFR, estimated glomerular filtration rate. 


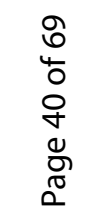




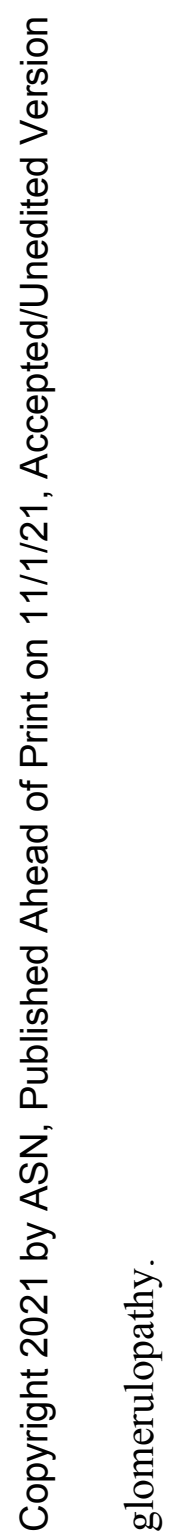




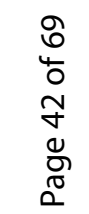

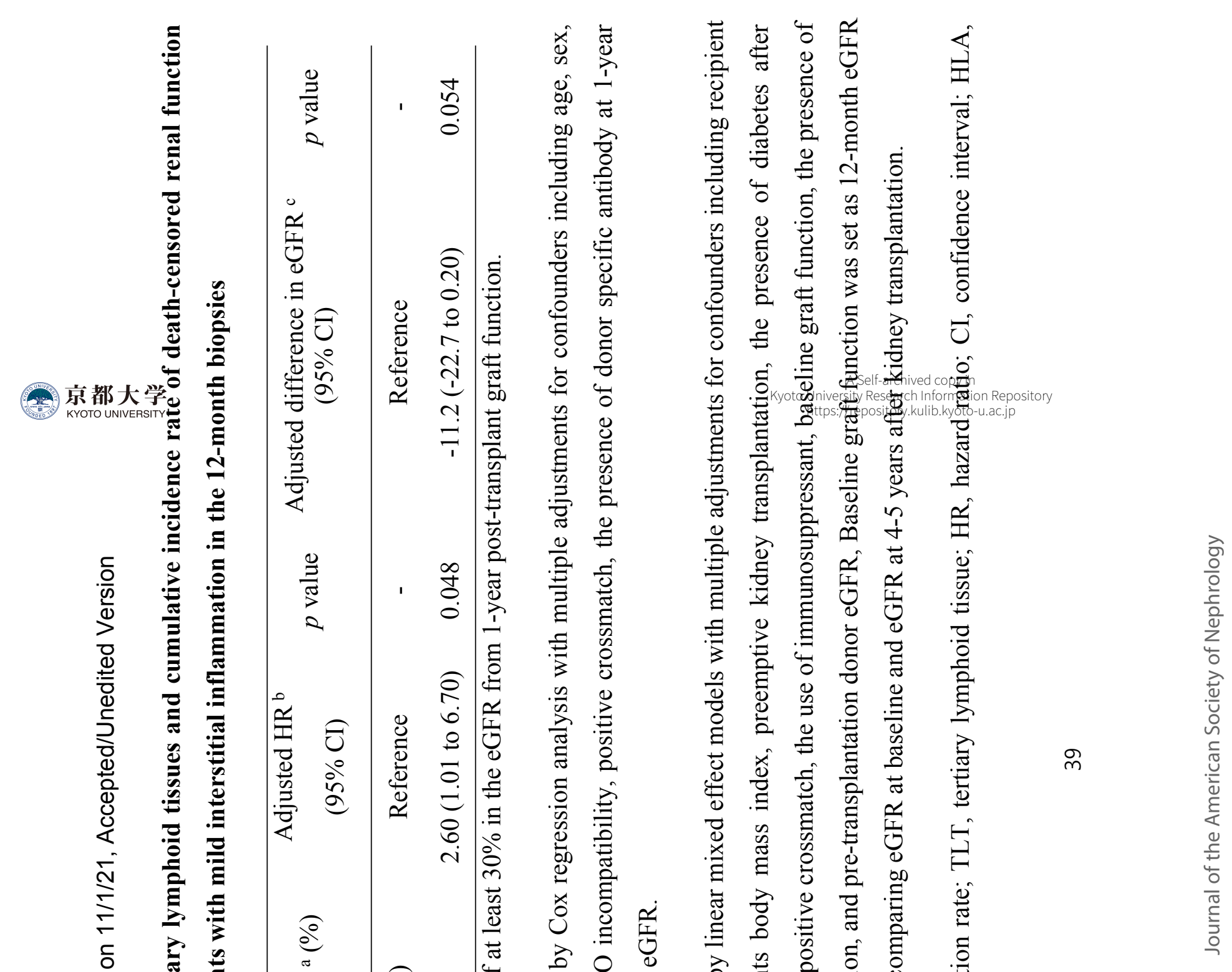


Copyright 2021 by ASN, Published Ahead of Print on 11/1/21, Accepted/Unedited Version

\section{Figure 1. A flowchart of the study participant selection}

Figure 1

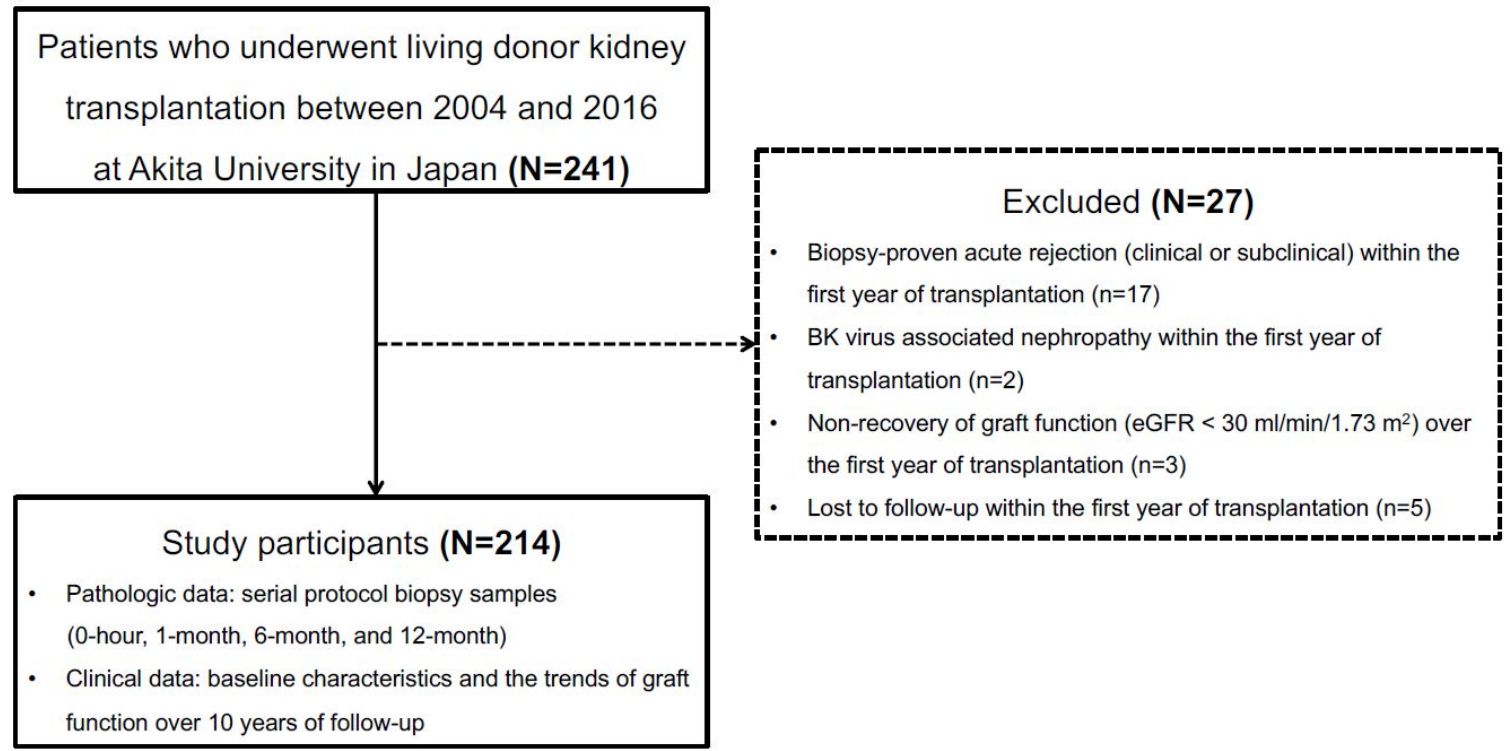

We retrospectively screened 241 patients who underwent living donor kidney transplantation between 2004 and 2016 at Akita University in Japan. After 27 patients with known risk factors for poor graft outcome and those lost to follow-up within a year of kidney transplantation were excluded, the remaining 214 kidney transplant recipients were enrolled. Serial protocol biopsy samples were obtained and processed for immunofluorescence to determine the presence and staging of tertiary lymphoid tissues.

eGFR, estimated glomerular filtration rate. 
Figure 2. Characterization of tertiary lymphoid tissues in transplanted kidney

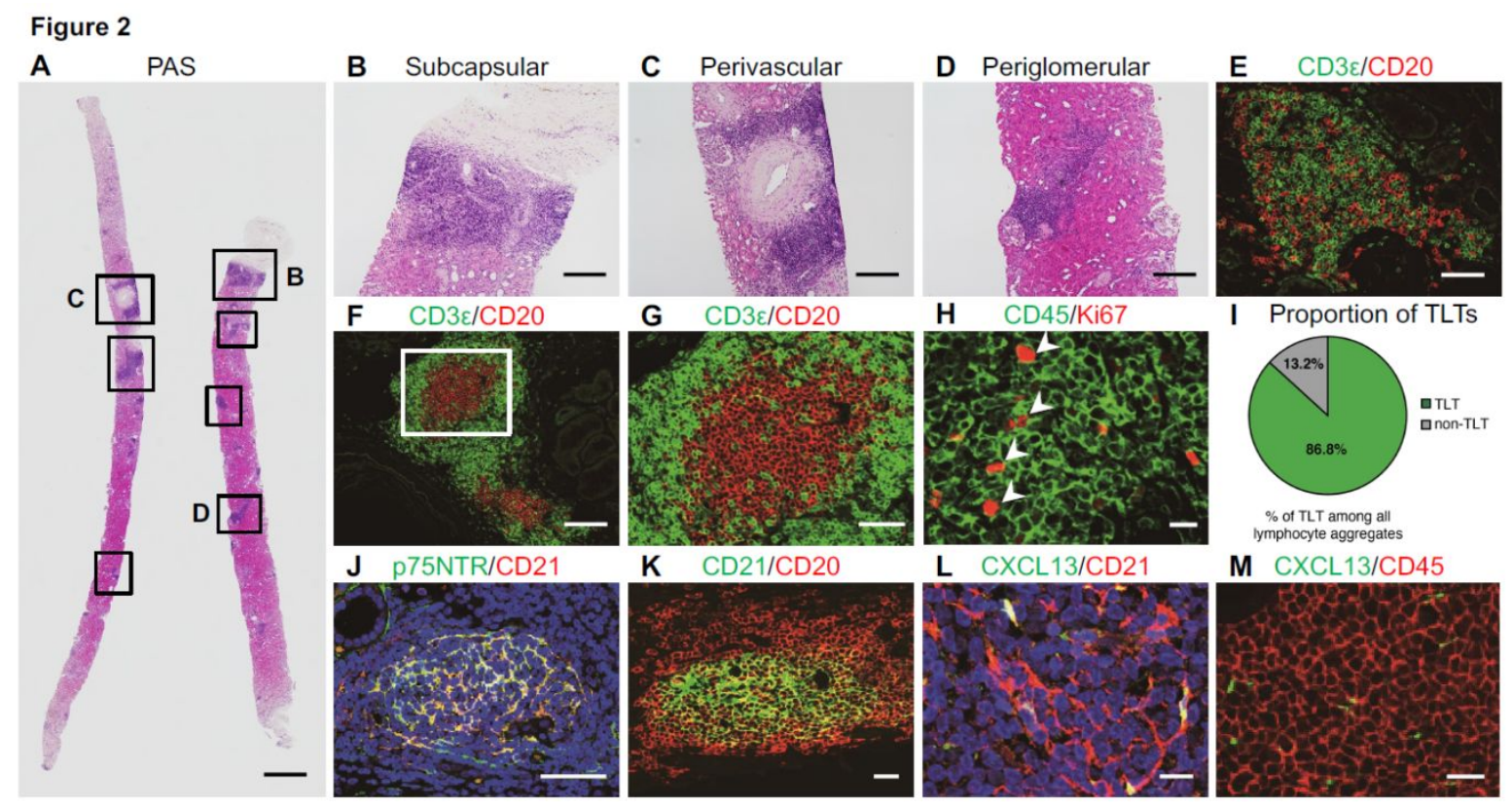

(A-D) Analyses of periodic acid-Schiff (PAS)-stained graft tissues revealing multiple lymphocyte infiltrates in protocol biopsy samples, as indicated by black boxes (A). The clusters were located either $(\mathbf{B})$ under the renal capsule, $(\mathbf{C})$ around blood vessels, or $(\mathbf{D})$ in the periglomerular area. (E-H) Immunofluorescence of $(\mathbf{E}, \mathbf{F}, \mathbf{G}) \mathrm{CD} 3 \varepsilon$ (a T cell marker) and CD20 (a B cell marker); and (H) CD45 (a common leukocyte marker) and Ki67 (a proliferation marker). (I) Proportion of tertiary lymphoid tissues among lymphocyte infiltrates found in PAS-stained samples. (J-M) Immunofluorescence of $(\mathbf{J})$ p75 neurotrophin receptor (p75NTR) and CD21; (K) CD21 and CD20; (L) CXCL13 and CD21; and (M) CXCL13 and CD45. Note that p75NTR and CD21 are expressed in follicular dendritic cells in tertiary lymphoid tissues, and CXCL13 is a main chemoattractant for B cells. Figure 1G shows a magnified view of the white box in Figure 1F. Arrowheads in Figure 1H indicate Ki67-positive proliferating lymphocytes. Scale bars; (A) $1 \mathrm{~mm}$; (B-D) $200 \mu \mathrm{m}$; (F) $100 \mu \mathrm{m}$; $(\mathbf{E}, \mathbf{G}, \mathbf{J}, \mathbf{K}) 50 \mu \mathrm{m} ;(\mathbf{H}, \mathbf{L}, \mathbf{M}) 10 \mu \mathrm{m}$. 
Copyright 2021 by ASN, Published Ahead of Print on 11/1/21, Accepted/Unedited Version

Figure 3. The staging of tertiary lymphoid tissues in transplanted kidney

Figure 3

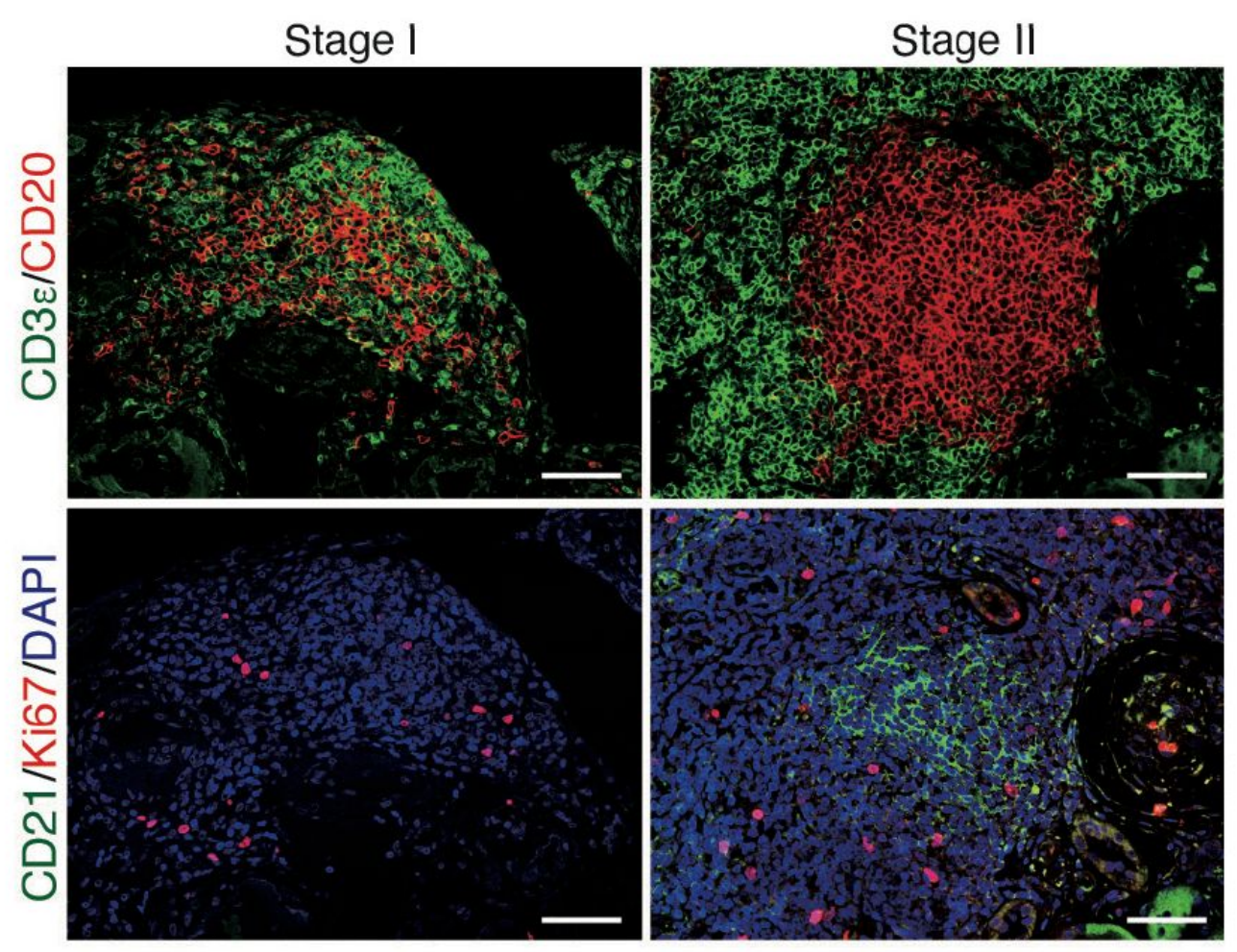

Representative immunofluorescence of different TLT stages as determined by the expression patterns of CD3e, CD20, CD21, and Ki67. Stage I TLTs were defined by the presence of lymphocyte clusters $\left(\mathrm{CD} 3 \varepsilon^{+}\right.$and $\left.\mathrm{CD} 20^{+}\right)$with signs of proliferation $\left(\mathrm{Ki} 67^{+}\right)$, and the absence of FDC (CD21-). Stage II TLTs were defined by the presence of lymphocyte clusters (CD3 $\varepsilon^{+}$ and $\left.\mathrm{CD} 20^{+}\right)$with signs of proliferation $\left(\mathrm{Ki}^{+} 7^{+}\right)$along with the presence of FDCs $\left(\mathrm{CD} 21^{+}\right)$.

TLTs, tertiary lymphoid tissues; FDCs, follicular dendritic cells.

Scale bars; $100 \mu \mathrm{m}$. 
Copyright 2021 by ASN, Published Ahead of Print on 11/1/21, Accepted/Unedited Version

Figure 4. The prevalence of tertiary lymphoid tissues in transplanted kidney

Figure 4

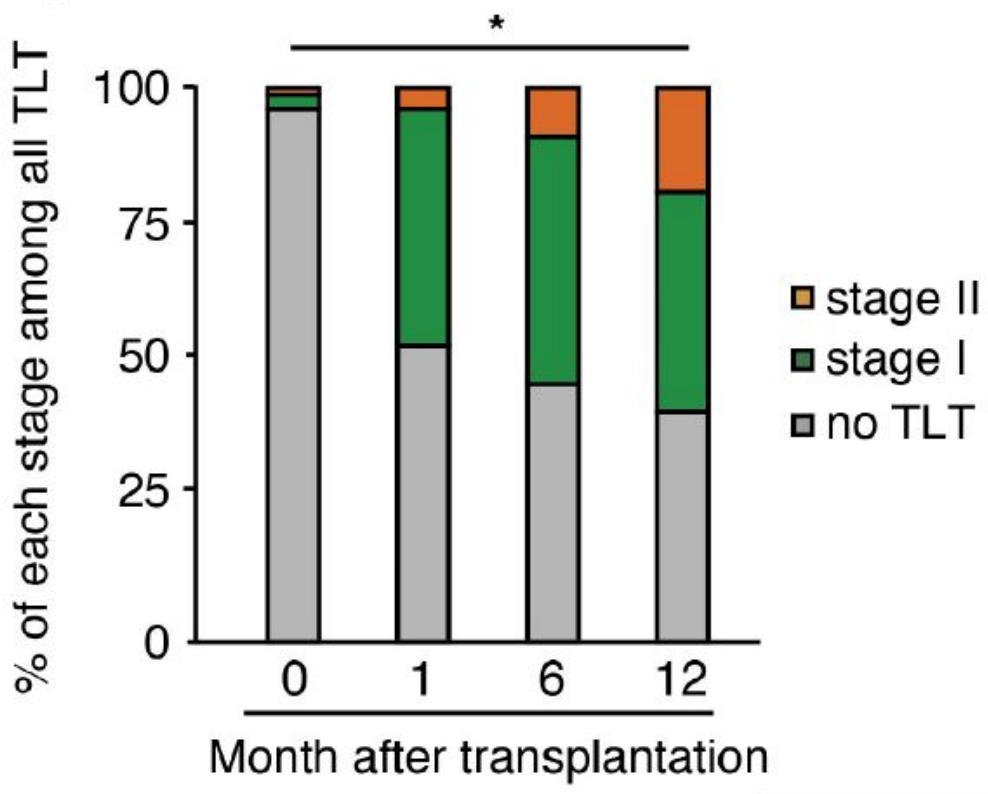

\begin{tabular}{ccccc|c|} 
No TLT & 202 & 102 & 76 & 77 & Number of \\
Stage I & 5 & 83 & 73 & 73 & patients \\
Stage II & 3 & 7 & 14 & 35 &
\end{tabular}

Relative frequency of patients with no TLT (gray), stage I TLTs (green), and stage II TLTs (orange) at various time points after kidney transplantation. The overall prevalence of TLTs increased from $3.8 \%$ at 0 -hour baseline to $46.9 \%$ at 1 month after kidney transplantation, and then slightly further during 12 months. By contrast, stage II TLTs exhibited a more gradual increase in prevalence, reaching $18.9 \%$ at 12 months post-transplantation. ${ }^{*} p<0.001$ by trends analysis

TLTs, tertiary lymphoid tissues. 
Figure 5. Renal allograft outcomes according to the staging of tertiary lymphoid tissues
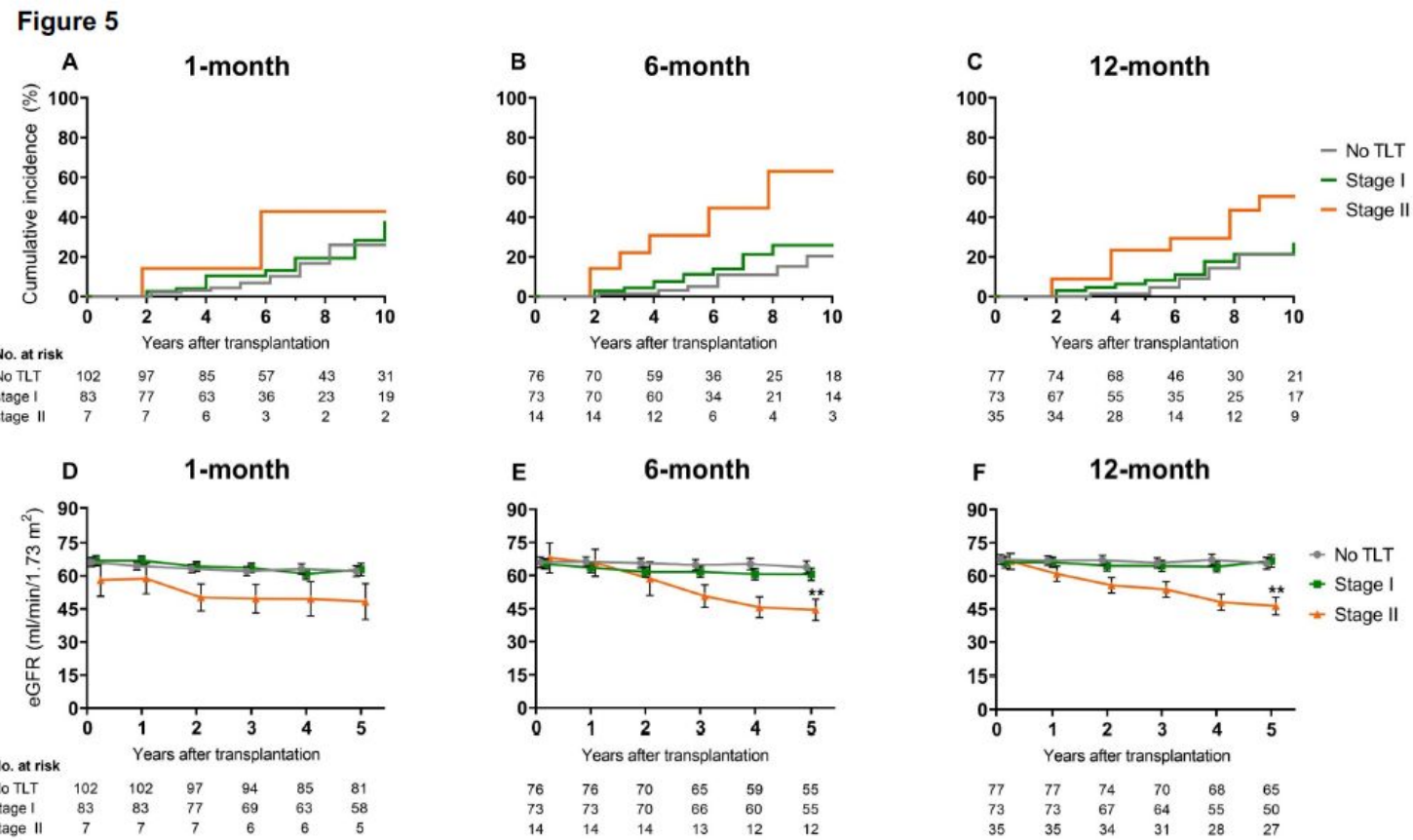
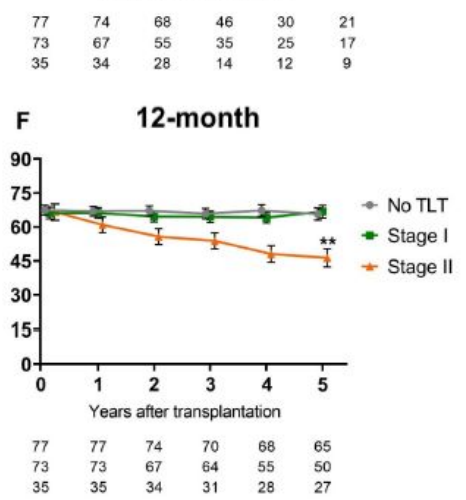

(A-C) The cumulative incidence rate of death-censored renal function decline and (D-F) the longitudinal trends of eGFR after kidney transplantation according to the stages of TLTs at given time points. One-month, 6-month, and 12-month refer to biopsy time points after kidney transplantation. Patients with stage II TLTs at 6- or 12-month experienced significantly accelerated graft dysfunction compared to those without TLT. The statistical comparisons between groups are performed by (A-C) Cox regression analysis and (D-F) linear mixed effect models with multiple adjustments, and their results are shown in Table 2 and 3, respectively. (D-F) Data are expressed as mean \pm standard error for each time point of follow-up. ${ }^{* *} p<0.005$, vs. No TLT. eGFR, estimated glomerular filtration rate; TLTs, tertiary lymphoid tissues. 
Copyright 2021 by ASN, Published Ahead of Print on 11/1/21, Accepted/Unedited Version

Figure 6. The effects of pre-transplantation rituximab on the prevalence of tertiary lymphoid tissues

Figure 6
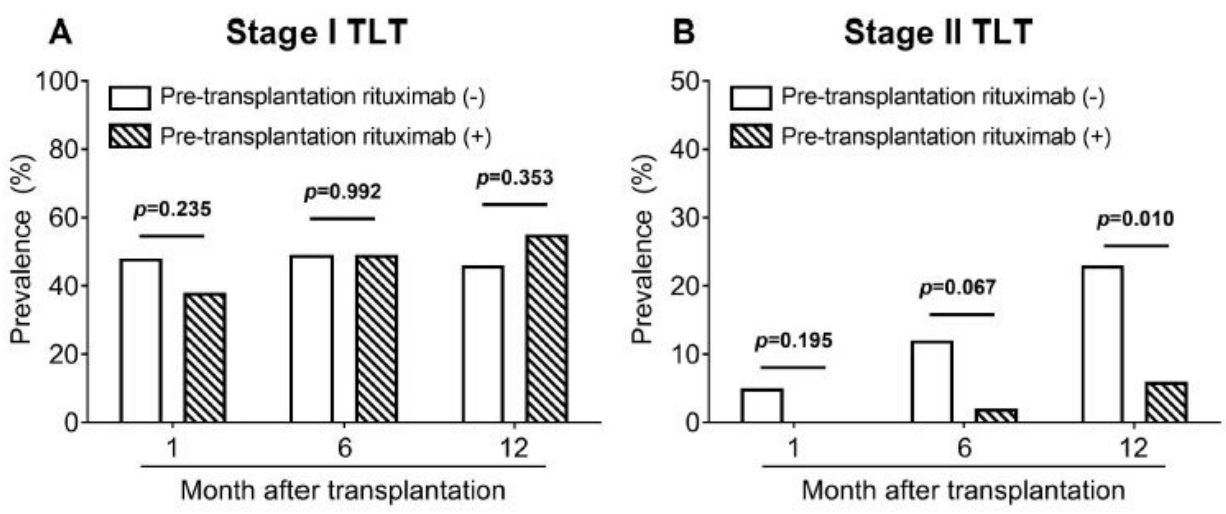

Prevalence of (A) stage I and (B) stage II TLTs at each time point of follow-up according to the use of pre-transplantation rituximab. The administration of rituximab before kidney transplantation was associated with a lower prevalence of stage II TLTs, but not with the change in stage I TLTs regardless of biopsy time point.

TLT, tertiary lymphoid tissues. 
Copyright 2021 by ASN, Published Ahead of Print on 11/1/21, Accepted/Unedited Version

Figure 7. Renal allograft outcomes according to the staging of tertiary lymphoid tissues among patients with mild interstitial inflammation in the 12-month biopsies

Figure 7
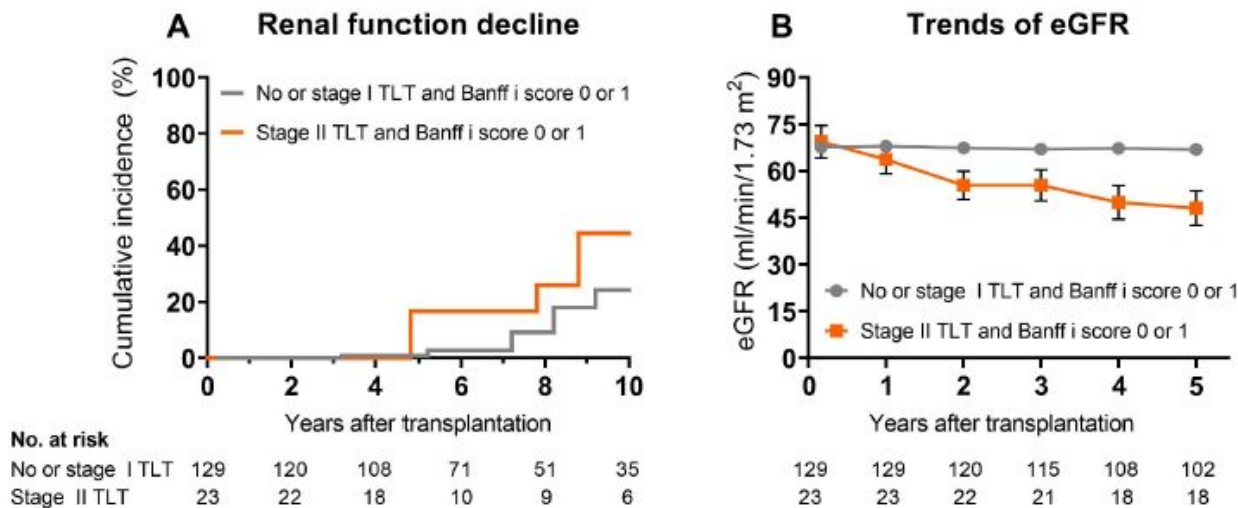

(A) The cumulative incidence rate of death-censored renal function decline and (B) eGFR over a period of 5 years after kidney transplantation according to the stages of TLTs in recipients with mild interstitial inflammation (Banff i score of 0 or 1). Patients with stage II TLTs in the 12-month biopsies experienced progressive declines in renal function even though the degree of overall interstitial inflammation was trivial. The statistical comparisons between groups are performed by (A) Cox regression analysis and (B) linear mixed effect models with multiple adjustments, and their results are shown in Table 6. (B) Data are expressed as mean \pm standard error for each time point of follow-up.

eGFR, estimated glomerular filtration rate; TLT, tertiary lymphoid tissues. 


\section{Advanced Tertiary Lymphoid Tissues in Protocol Biopsies are Associated with Progressive Graft Dysfunction in Kidney Transplant Recipients}

\section{METHODS}

Longitudinal retrospective cohort Recruited in 2004 2016

\section{Study participants $(\mathrm{N}=\mathbf{2 1 4})$}

$\checkmark$ Living donor-related kidney transplant

$\checkmark$ No clinical or subclinical rejection within first year after transplantation

Data acquisitions

$\checkmark$ Protocol biopsy samples (obtained at 0-, 1-, 6 , and 12-month post-transplantation)

$\checkmark 5$-year trends of renal allograft function

Tertiary lymphoid tissue (TLT) staging

$\checkmark$ Stage l: presence of lymphocyte aggregates with signs of proliferation

Stage II: presence of follicular dendritic cells

\section{OUTCOME}

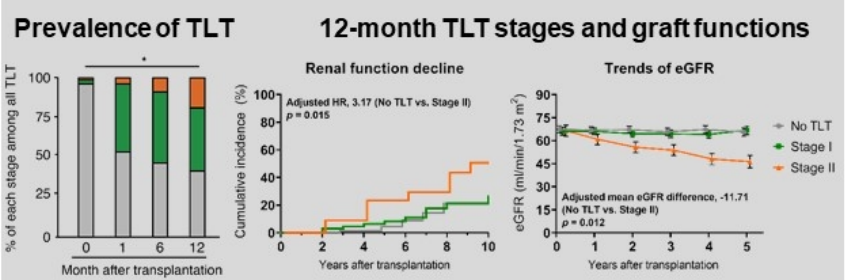

Pre-transplant rituximab

TLT stages and Banff scores

\begin{tabular}{|c|c|c|c|c|c|}
\hline \multirow[b]{2}{*}{ Stage II TLT $\downarrow$} & \multirow{2}{*}{$\begin{array}{c}\text { 12-month } \\
\text { Banff score }\end{array}$} & \multicolumn{3}{|c|}{ 12-month biopsies } & \multirow{2}{*}{$\begin{array}{c}p \text { value } \\
\text { (No TLT vs. } \\
\text { stage II) }\end{array}$} \\
\hline & & No TLT & Stage I & Stage II & \\
\hline Odd ratio: 0.17 & $t$ & $0.12 \pm 0.36$ & $0.38 \pm 0.68$ & $0.60 \pm 0.91$ & $<0.001$ \\
\hline \multirow{2}{*}{$(95 \% \mathrm{Cl} 0.04-0.72)$} & ct & $0.77 \pm 0.72$ & $1.01 \pm 0.62$ & $1.29=0.75$ & $<0.001$ \\
\hline & ci & $0.70 \pm 0.63$ & $0.99 \pm 0.68$ & $1.17 \pm 0.92$ & 0.008 \\
\hline
\end{tabular}

Conclusion

doi: 10.1681/ASN.2021050715

TLTs are commonly found in clinically stable transplanted kidneys and advanced stage II TLTs

are associated with progressive graft dysfunction after kidney transplantation.

254x190mm (96 x 96 DPI) 


\section{SUPPLEMENTAL APPENDIX FOR THE STUDY:}

Advanced tertiary lymphoid tissues in protocol biopsies are associated with progressive graft dysfunction in kidney transplant recipients 


\section{Table of contents}

Supplemental Table 1. Baseline characteristics and clinical parameters of patients according to $\mathrm{ABO}$ incompatibility

Supplemental Table 2. Baseline characteristics, clinical parameters, and pathologic classifications of patients who experienced acute rejection within the first year posttransplantation

Supplemental Table 3. Hazard ratios of the stages of tertiary lymphoid tissues for deathcensored renal function decline in subgroup of recipients who underwent ABO-compatible kidney transplantation

Supplemental Table 4. Association between the stages of tertiary lymphoid tissues and graft function in subgroup of recipients who underwent $\mathrm{ABO}$-compatible kidney transplantation

Supplemental Table 5. Multivariable analyses of risk factors for the development of stage I tertiary lymphoid tissues in 12-month biopsies

Supplemental Table 6. The stages of tertiary lymphoid tissues according to ABO compatibility Supplemental Table 7. Banff pathologic score at 12-month post-transplantation according to ABO compatibility

Supplemental Figure 1. Variations in the sizes of tertiary lymphoid tissues in transplanted kidneys

Supplemental Figure 2. T cell-dominant tertiary lymphoid tissues in transplanted kidneys treated with pre-transplantation rituximab

Supplemental Figure 3. Renal allograft outcomes according to the presence of tertiary lymphoid tissues

Supplemental Figure 4. Renal allograft outcomes according to the stages of tertiary lymphoid tissues in subgroup of recipients who underwent ABO-compatible kidney transplantation

Supplemental Figure 5. The prevalence of donor specific antibody at 12 months after kidney transplantation according to the stages of tertiary lymphoid tissues

Supplemental Figure 6. Longitudinal trends of renal allograft function according to the history of borderline $\mathrm{T}$ cell-mediated rejection within the first year after kidney transplantation

Supplemental Figure 7. Longitudinal trends of renal allograft function according to the use of pre-transplantation rituximab and the stages of tertiary lymphoid tissues in the 12-month biopsies

Supplemental Figure 8. Association between the stages of tertiary lymphoid tissues and interstitial inflammation scores 


\section{Supplemental Table 1. Baseline characteristics and clinical parameters of patients according to ABO incompatibility}

\begin{tabular}{|c|c|c|c|}
\hline & $\begin{array}{c}\text { ABO- } \\
\text { compatible }\end{array}$ & $\begin{array}{c}\text { ABO- } \\
\text { incompatible }\end{array}$ & $p$ value \\
\hline Number of patients & 161 & 53 & \\
\hline Age (year) & $47.0 \pm 12.7$ & $54.4 \pm 10.1$ & $<0.001$ \\
\hline Gender (Male, \%) & $106(65.8)$ & $33(62.3)$ & 0.636 \\
\hline Body mass index $\left(\mathrm{kg} / \mathrm{m}^{2}\right)$ & $22.5 \pm 3.7$ & $22.1 \pm 3.8$ & 0.487 \\
\hline \multicolumn{4}{|l|}{ Etiology of end-stage renal disease (n, \%) } \\
\hline Chronic glomerulonephritis & $91(56.5)$ & $31(58.5)$ & \multirow{5}{*}{0.902} \\
\hline Diabetes mellitus & $28(17.4)$ & $8(15.1)$ & \\
\hline Hypertension & $11(6.8)$ & $5(9.4)$ & \\
\hline Polycystic kidney disease & $10(6.2)$ & $4(7.5)$ & \\
\hline Others ${ }^{\text {a }}$ & $21(13.0)$ & $5(9.5)$ & \\
\hline Time on dialysis (month) & $17.0[4.0,49.0]$ & $21.0[5.5,51.5]$ & 0.346 \\
\hline Preemptive kidney transplantation (n, \%) & $33(20.5)$ & $7(13.2)$ & 0.238 \\
\hline Number of HLA mismatching (n) & $3.2 \pm 1.5$ & $3.5 \pm 1.4$ & 0.139 \\
\hline Positive crossmatch (n, \%) & $10(6.2)$ & $4(7.5)$ & 0.752 \\
\hline Pre-transplantation rituximab (n, \%) & $7(4.3)$ & $50(94.3)$ & $<0.001$ \\
\hline Cold ischemic time (minute) & $142.7 \pm 33.0$ & $147.9 \pm 49.2$ & 0.388 \\
\hline Warm ischemic time (minute) & $5.1 \pm 4.2$ & $4.8 \pm 1.4$ & 0.600 \\
\hline \multicolumn{4}{|l|}{ Induction immunosuppressant (n, \%) } \\
\hline Basiliximab & $161(100)$ & $53(100)$ & 1.000 \\
\hline \multicolumn{4}{|l|}{ Maintenance immunosuppressant ${ }^{b}(n, \%)$} \\
\hline Prednisolone & $127(78.9)$ & $44(83.0)$ & 0.514 \\
\hline Tacrolimus & $161(100)$ & $53(100)$ & 1.000 \\
\hline Mycophenolate mofetil & $161(100)$ & $53(100)$ & 1.000 \\
\hline 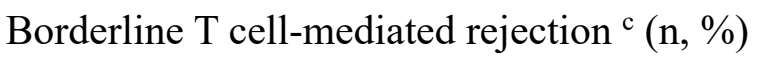 & $48(29.8)$ & $18(34.0)$ & 0.571 \\
\hline $\begin{array}{l}\text { Donor specific antibody at } 1 \text {-year post- } \\
\text { transplantation }(n, \%)^{d}\end{array}$ & $10 / 158(6.3)$ & $3 / 49(6.1)$ & 1.000 \\
\hline Class I & $5(50.0)$ & $2(66.7)$ & 1.000 \\
\hline Class II & $5(50.0)$ & $2(66.7)$ & 1.000 \\
\hline
\end{tabular}




\begin{tabular}{lccc}
\hline Posttransplant eGFR $\left(\mathrm{ml} / \mathrm{min} / 1.73 \mathrm{~m}^{2}\right)$ & & & \\
$\quad$ 1-month & $65.1 \pm 20.7$ & $69.7 \pm 20.0$ & 0.155 \\
$\quad$ 1-year & $65.1 \pm 18.9$ & $65.4 \pm 18.6$ & 0.918 \\
$\quad$ 5-year & $61.2 \pm 22.3$ & $65.9 \pm 18.7$ & 0.262 \\
Donor age (year) & $58.1 \pm 9.9$ & $59.2 \pm 9.7$ & 0.453 \\
Donor gender (Male, \%) & $62(38.5)$ & $20(37.7)$ & 0.920 \\
Pre-transplantation donor eGFR $\left(\mathrm{ml} / \mathrm{min} / 1.73 \mathrm{~m}^{2}\right)$ & $103.1 \pm 10.8$ & $102.6 \pm 10.8$ & 0.743 \\
\hline
\end{tabular}

Data were expressed as mean \pm standard deviation or the number of patients (percentage), and were compared by independent t-tests, chi-square tests, or Fisher's exact tests. Time on dialysis was described as median [ $\left[1^{\text {st }}\right.$ and $3^{\text {rd }}$ interquartile range] and was compared by Mann-Whitney test because it was non-normally distributed.

${ }^{a}$ Others include chronic tubulointerstitial nephritis, gestational hypertension, vesicoureteral reflux disease, sepsis, cystinuria, and bone marrow transplant nephropathy.

${ }^{b}$ Data obtained at the time of outpatient visit 1 year after kidney transplantation.

${ }^{c}$ At least one episode during the first year after transplantation.

${ }^{\mathrm{d}}$ Not assessed in 7 recipients.

Abbreviation: HLA, human leukocyte antigen; eGFR, estimated glomerular filtration rate. 


\section{Supplemental Table 2. Baseline characteristics, clinical parameters, and pathologic classifications of patients who experienced acute rejection within the first year post- transplantation}

\begin{tabular}{|c|c|}
\hline Number of patients & 17 \\
\hline Age (year) & $50.6 \pm 8.2$ \\
\hline Gender (Male, \%) & $8(47.1)$ \\
\hline Body mass index $\left(\mathrm{kg} / \mathrm{m}^{2}\right)$ & $23.2 \pm 5.0$ \\
\hline \multicolumn{2}{|l|}{ Etiology of end-stage renal disease (n, \%) } \\
\hline Chronic glomerulonephritis & $14(82.4)$ \\
\hline Diabetes mellitus & $2(11.8)$ \\
\hline Polycystic kidney disease & $1(5.9)$ \\
\hline Time on dialysis (month) & $26.0[12.0,54.0]$ \\
\hline Preemptive kidney transplantation (n, \%) & $3(17.6)$ \\
\hline Number of HLA mismatching (n) & $3.3 \pm 1.5$ \\
\hline Positive crossmatch $(\mathrm{n}, \%)$ & $4(23.5)$ \\
\hline ABO-incompatible kidney transplantation (n, \%) & $6(35.3)$ \\
\hline Pre-transplantation rituximab (n, \%) & $9(52.9)$ \\
\hline Cold ischemic time (minute) & $171.6 \pm 40.6$ \\
\hline Warm ischemic time (minute) & $4.5 \pm 1.7$ \\
\hline \multicolumn{2}{|l|}{ Induction immunosuppressant (n, \%) } \\
\hline Basiliximab & $17(100)$ \\
\hline \multicolumn{2}{|l|}{ Maintenance immunosuppressant ${ }^{\mathrm{a}}(\mathrm{n}, \%)$} \\
\hline Prednisolone & $15(88.2)$ \\
\hline Tacrolimus & $17(100)$ \\
\hline Mycophenolate mofetil & $17(100)$ \\
\hline Donor specific antibody at 1 -year post-transplantation (n, \%) & $5(29.4)$ \\
\hline Class I & $4(23.5)$ \\
\hline Class II & $2(11.8)$ \\
\hline \multicolumn{2}{|l|}{ Posttransplant eGFR (ml/min/1.73 $\left.\mathrm{m}^{2}\right)$} \\
\hline 1-month & $58.0 \pm 17.1$ \\
\hline 1-year & $54.3 \pm 24.0$ \\
\hline 5 -year & $55.5 \pm 18.3$ \\
\hline
\end{tabular}




\begin{tabular}{lc}
\hline Donor age (year) & $56.2 \pm 15.0$ \\
Donor gender (Male, \%) & $9(52.9)$ \\
Pre-transplantation donor eGFR $\left(\mathrm{ml} / \mathrm{min} / 1.73 \mathrm{~m}^{2}\right)$ & $101.1 \pm 17.2$ \\
Rejection type (n, \%) & \\
$\quad$ Acute T cell-mediated rejection, IA & $3(17.6)$ \\
$\quad$ Acute T cell-mediated rejection, IB & $1(5.9)$ \\
$\quad$ Acute antibody-mediated rejection & $13(76.5)$
\end{tabular}

Data were expressed as mean \pm standard deviation or the number of patients (percentage). Time on dialysis was described as median [ $1^{\text {st }}$ and $3^{\text {rd }}$ interquartile range].

${ }^{a}$ Data obtained at the time of outpatient visit 1 year after transplantation.

Abbreviation: HLA, human leukocyte antigen; eGFR, estimated glomerular filtration rate. 


\section{Supplemental Table 3. Hazard ratios of the stages of tertiary lymphoid tissues for death- censored renal function decline in subgroup of recipients who underwent ABO- compatible kidney transplantation}

\begin{tabular}{ccccc}
\hline $\begin{array}{c}\text { Biopsy } \\
\text { time point }\end{array}$ & TLT stages & No. of events ${ }^{\mathrm{a}}(\%)$ & $\begin{array}{c}\text { Adjusted HR } \\
(95 \% \text { CI })\end{array}$ & $p$ value \\
\hline \multirow{2}{*}{ 1-month } & No TLT $(\mathrm{n}=72)$ & $13(18.1)$ & Reference & - \\
& Stage I $(\mathrm{n}=62)$ & $14(22.6)$ & $1.24(0.57$ to 2.71) & 0.594 \\
& Stage II $(\mathrm{n}=7)$ & $3(42.9)$ & $3.15(0.82$ to 12.09$)$ & 0.094 \\
\hline \multirow{2}{*}{ 6-month } & No TLT $(\mathrm{n}=55)$ & $7(12.7)$ & Reference & - \\
& Stage I $(\mathrm{n}=51)$ & $10(19.6)$ & $1.12(0.40$ to 3.16) & 0.827 \\
& Stage II $(\mathrm{n}=13)$ & $6(46.2)$ & $3.97(1.15$ to 13.76$)$ & 0.030 \\
\hline \multirow{2}{*}{ 12-month } & No TLT $(\mathrm{n}=60)$ & $10(16.7)$ & Reference & - \\
& Stage I $(\mathrm{n}=49)$ & $7(14.3)$ & $0.72(0.25$ to 2.11) & 0.723 \\
& Stage II $(\mathrm{n}=33)$ & $14(42.4)$ & $2.81(1.05$ to 7.51$)$ & 0.039 \\
\hline
\end{tabular}

${ }^{a}$ Renal outcome was defined as a decline of at least $30 \%$ in the eGFR from 1-year posttransplant graft function.

$\mathrm{b}$ The comparisons between groups are performed by Cox regression analysis with multiple adjustments for confounders including age, sex, the presence of diabetes after transplantation, positive crossmatch, the presence of donor specific antibody at 1-year post-transplantation, and pre-transplantation donor eGFR.

Abbreviation: TLT, tertiary lymphoid tissue; HR, hazard ratio; CI, confidence interval; eGFR, estimated glomerular filtration rate. 


\section{Supplemental Table 4. Association between the stages of tertiary lymphoid tissues and graft function in subgroup of recipients who underwent ABO-compatible kidney transplantation}

\begin{tabular}{cccc}
\hline \multirow{2}{*}{ Biopsy time point } & TLT stages & $\begin{array}{c}\text { Adjusted difference in eGFR } \\
(95 \% \text { CI })\end{array}$ & $p$ value \\
\hline \multirow{2}{*}{ 1-month } & No TLT $(\mathrm{n}=72)$ & Reference & - \\
& Stage I $(\mathrm{n}=62)$ & $3.03(-3.45$ to 9.50$)$ & 0.359 \\
& Stage II $(\mathrm{n}=7)$ & $-12.04(-31.11$ to 7.04$)$ & 0.216 \\
\hline \multirow{3}{*}{ 6-month } & No TLT $(\mathrm{n}=55)$ & Reference & - \\
& Stage I $(\mathrm{n}=51)$ & $-2.92(-9.89$ to 4.05$)$ & 0.412 \\
& Stage II $(\mathrm{n}=13)$ & $-14.18(-25.67$ to -2.69$)$ & 0.016 \\
\hline \multirow{2}{*}{ 12-month } & No TLT $(\mathrm{n}=60)$ & Reference & - \\
& Stage I $(\mathrm{n}=49)$ & $-1.75(-7.12$ to 3.61$)$ & 0.522 \\
& Stage II $(\mathrm{n}=33)$ & $-13.26(-23.26$ to -3.25$)$ & 0.009 \\
\hline
\end{tabular}

a The comparisons between groups are performed by linear mixed effect models with multiple adjustments for confounders including recipient age and gender, donor age and gender, recipients body mass index, preemptive kidney transplantation, the presence of diabetes after transplantation, the number of HLA mismatching, positive crossmatch, the use of immunosuppressant, baseline graft function, the presence of donor specific antibody at 1-year post-transplantation, and pre-transplantation donor eGFR. Baseline graft function was set as eGFR levels for each time point. The differences in eGFR are calculated by comparing eGFR at baseline and eGFR at 4-5 years after kidney transplantation.

Abbreviation: TLT, tertiary lymphoid tissue; eGFR, estimated glomerular filtration rate; CI, confidence interval; HLA, human leukocyte antigen. 
Supplemental Table 5. Multivariable analyses of risk factors for the development of stage I tertiary lymphoid tissues in 12-month biopsies

\begin{tabular}{|c|c|c|}
\hline Variables & OR $(95 \% \mathrm{CI})$ & $p$ value \\
\hline Recipient age (per 10-year increase) & $1.08(0.79-1.48)$ & 0.631 \\
\hline Donor age (per 10-year increase) & $0.69(0.57-1.46)$ & 0.688 \\
\hline Recipient gender (male) & $2.09(0.86-5.06)$ & 0.103 \\
\hline Donor gender (male) & $0.82(0.37-1.82)$ & 0.622 \\
\hline Body mass index (per $1 \mathrm{~kg} / \mathrm{m}^{2}$ increase) & $1.07(0.97-1.19)$ & 0.191 \\
\hline Diabetes mellitus & $1.20(0.53-2.68)$ & 0.665 \\
\hline Number of HLA mismatching (per one mismatch increase) & $0.96(0.74-1.25)$ & 0.767 \\
\hline Positive crossmatch & $0.10(0.01-1.01)$ & 0.051 \\
\hline Pre-transplantation rituximab & $1.76(0.81-3.80)$ & 0.151 \\
\hline Steroid maintenance therapy at 1 -year post-transplantation & $2.62(1.01-6.82)$ & 0.048 \\
\hline Cold ischemic time (per ten-minute increase) & $1.08(1.01-1.15)$ & 0.031 \\
\hline Warm ischemic time (per an minute increase) & $1.08(0.97-1.20)$ & 0.173 \\
\hline Donor specific antibody at 1 -year post-transplantation & $0.34(0.06-2.10)$ & 0.246 \\
\hline 12-month eGFR (per $10 \mathrm{ml} / \mathrm{min} / 1.73 \mathrm{~m}^{2}$ increase) & $0.96(0.77-1.21)$ & 0.729 \\
\hline Donor eGFR (per $10 \mathrm{ml} / \mathrm{min} / 1.73 \mathrm{~m}^{2}$ increase) & $1.33(0.86-2.08)$ & 0.203 \\
\hline Borderline T cell-mediated rejection & $2.05(0.97-4.34)$ & 0.060 \\
\hline
\end{tabular}

ABO incompatibility was not used as variables because of its significant correlation with the use of pre-transplantation rituximab.

Abbreviation: OR, odds ratio; CI, confidence interval; HLA, human leukocyte antigen; eGFR, estimated glomerular filtration rate. 


\section{Supplemental Table 6. The stages of tertiary lymphoid tissues according to ABO compatibility}

\begin{tabular}{ccccc}
\hline & TLT stages & ABO-compatible & ABO-incompatible & $p$ value \\
\hline \multirow{2}{*}{1 -month } & No TLT & $72(51.1)$ & $30(58.8)$ & \\
$(\mathrm{n}=192)$ & Stage I & $62(44.0)$ & $21(41.2)$ & 0.172 \\
& Stage II & $7(5.0)$ & $0(0)$ & \\
\hline \multirow{2}{*}{6 -month } & No TLT & $55(46.2)$ & $21(47.7)$ & 0.368 \\
$(\mathrm{n}=163)$ & Stage I & $51(42.9)$ & $22(50.0)$ & \\
& Stage II & $13(10.9)$ & $1(2.3)$ & 0.222 \\
$12-$-month & No TLT & $60(42.3)$ & $17(39.5)$ & \\
$(\mathrm{n}=185)$ & Stage I & $49(34.5)$ & $24(55.8)$ & $2(4.7)$ \\
\hline
\end{tabular}

Data were expressed as the number of patients (percentage), and were compared by trend analysis.

Abbreviation: TLT, tertiary lymphoid tissue. 
Supplemental Table 7. Banff pathologic score at 12-month post-transplantation according to $\mathrm{ABO}$ compatibility

\begin{tabular}{cccc}
\hline 12-month Banff scores & ABO-compatible & ABO-incompatible & $p$ value \\
\hline $\mathrm{i}$ & $0.68 \pm 0.83$ & $0.86 \pm 0.81$ & 0.104 \\
$\mathrm{t}$ & $0.27 \pm 0.61$ & $0.40 \pm 0.67$ & 0.091 \\
$\mathrm{v}$ & $0 \pm 0$ & $0 \pm 0$ & 1.000 \\
$\mathrm{~g}$ & $0.01 \pm 0.11$ & $0.02 \pm 0.14$ & 0.697 \\
$\mathrm{ptc}$ & $0.11 \pm 0.35$ & $0.10 \pm 0.36$ & 0.782 \\
$\mathrm{ct}$ & $0.93 \pm 0.69$ & $1.04 \pm 0.70$ & 0.282 \\
$\mathrm{ci}$ & $0.81 \pm 0.74$ & $0.96 \pm 0.70$ & 0.150 \\
$\mathrm{cv}$ & $0.02 \pm 0.24$ & $0 \pm 0$ & 0.576 \\
$\mathrm{cg}$ & $0 \pm 0$ & $0 \pm 0$ & 1.000 \\
$\mathrm{C} 4 \mathrm{~d}$ & $0.27 \pm 0.65$ & $1.93 \pm 1.09$ & $<0.001$ \\
\hline
\end{tabular}

Data were expressed as mean \pm standard deviation, and were compared by Kruskal-Wallis test. Abbreviation: i, interstitial inflammation; $t$, tubulitis; $v$, intimal arteritis; g, glomerulitis; ptc, peritubular capillaritis; ct, tubular atrophy; ci, interstitial fibrosis; cv, chronic fibrous intimal thickening; cg, transplant glomerulopathy. 
Supplemental Figure 1. Variations in the sizes of tertiary lymphoid tissues in transplanted kidneys

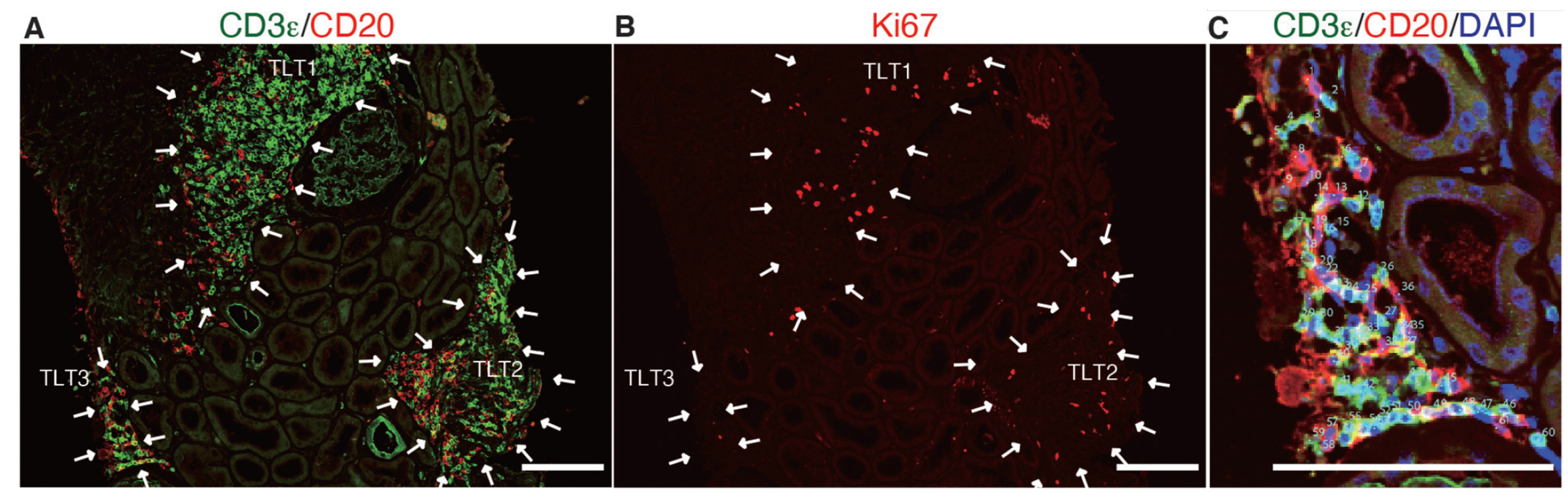

Immunofluorescence of (A, C) CD3 $\varepsilon$ and CD20 and (B) Ki67 in transplanted kidneys. Supplementary Figure 1C shows a magnified view of TLT3 in Supplementary Figure 1A. Scale bars: $100 \mu \mathrm{m}$.

Abbreviation: TLT, tertiary lymphoid tissues. 
Supplemental Figure 2. T cell-dominant tertiary lymphoid tissues in transplanted kidneys treated with pre-transplantation rituximab

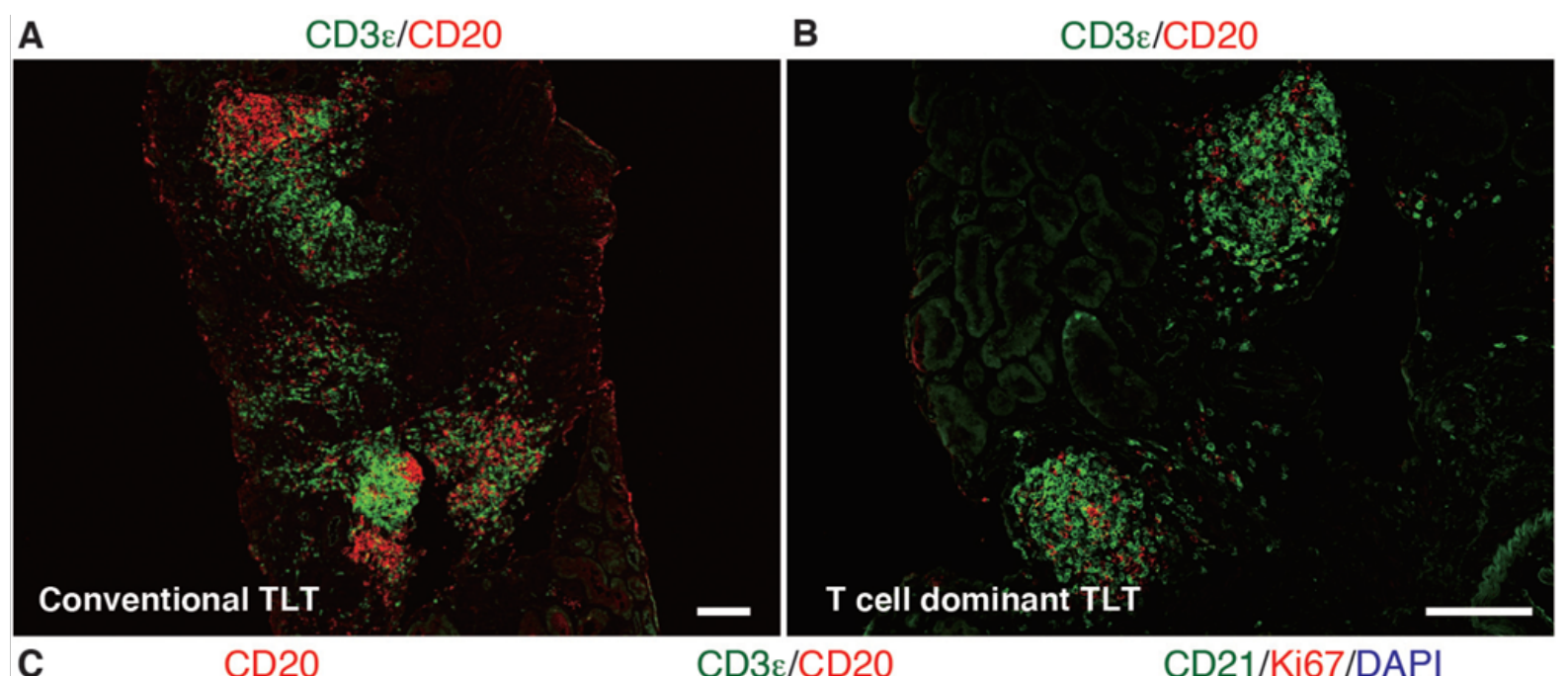

C

$\mathrm{CD} 3 \varepsilon / \mathrm{CD} 20$

CD21/Ki67/DAPI
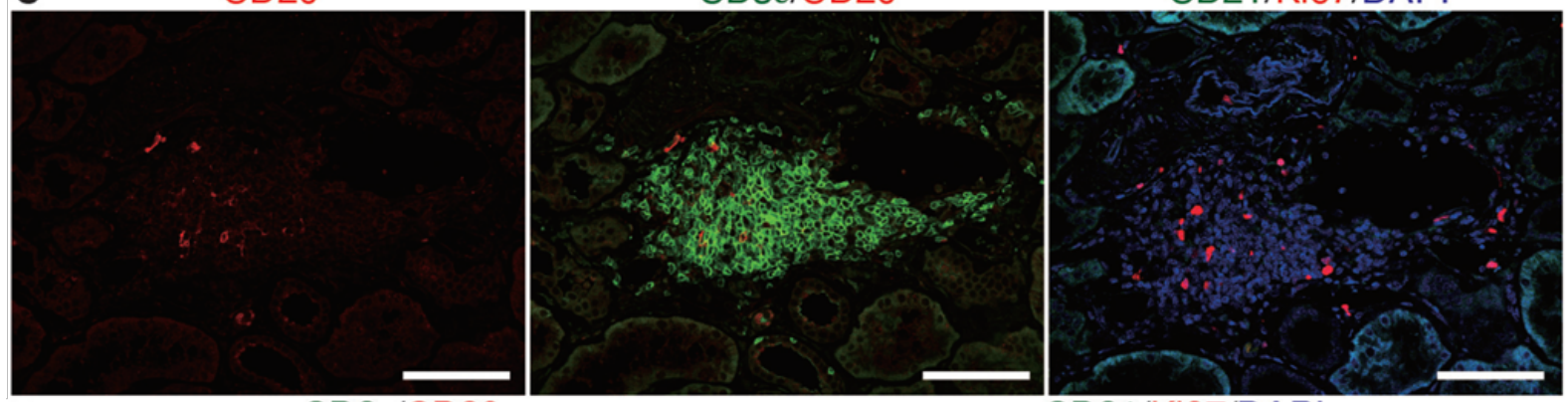

D

$\mathrm{CD} 3 \varepsilon / \mathrm{CD} 20$

CD21/Ki67/DAPI

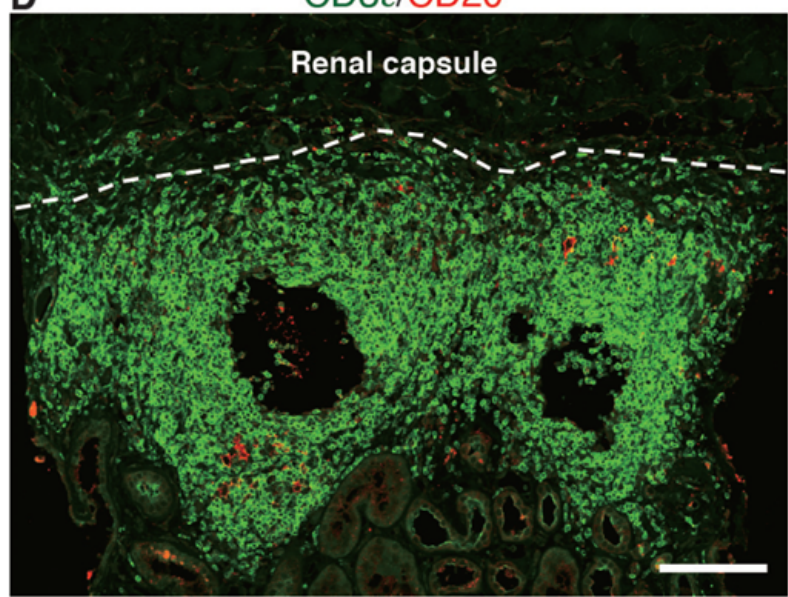

Renal capsule

(A-D) Immunofluorescence of (A-B) CD3 $\varepsilon$ and CD20 and (C-D) CD3 $\varepsilon$ and CD20, CD21 and Ki67 in transplanted kidneys. Serial sections were stained in (C-D). Scale bars: (A, B, D) 100 $\mu \mathrm{m},(\mathbf{C}) 50 \mu \mathrm{m}$.

Abbreviation: TLT, tertiary lymphoid tissues. 

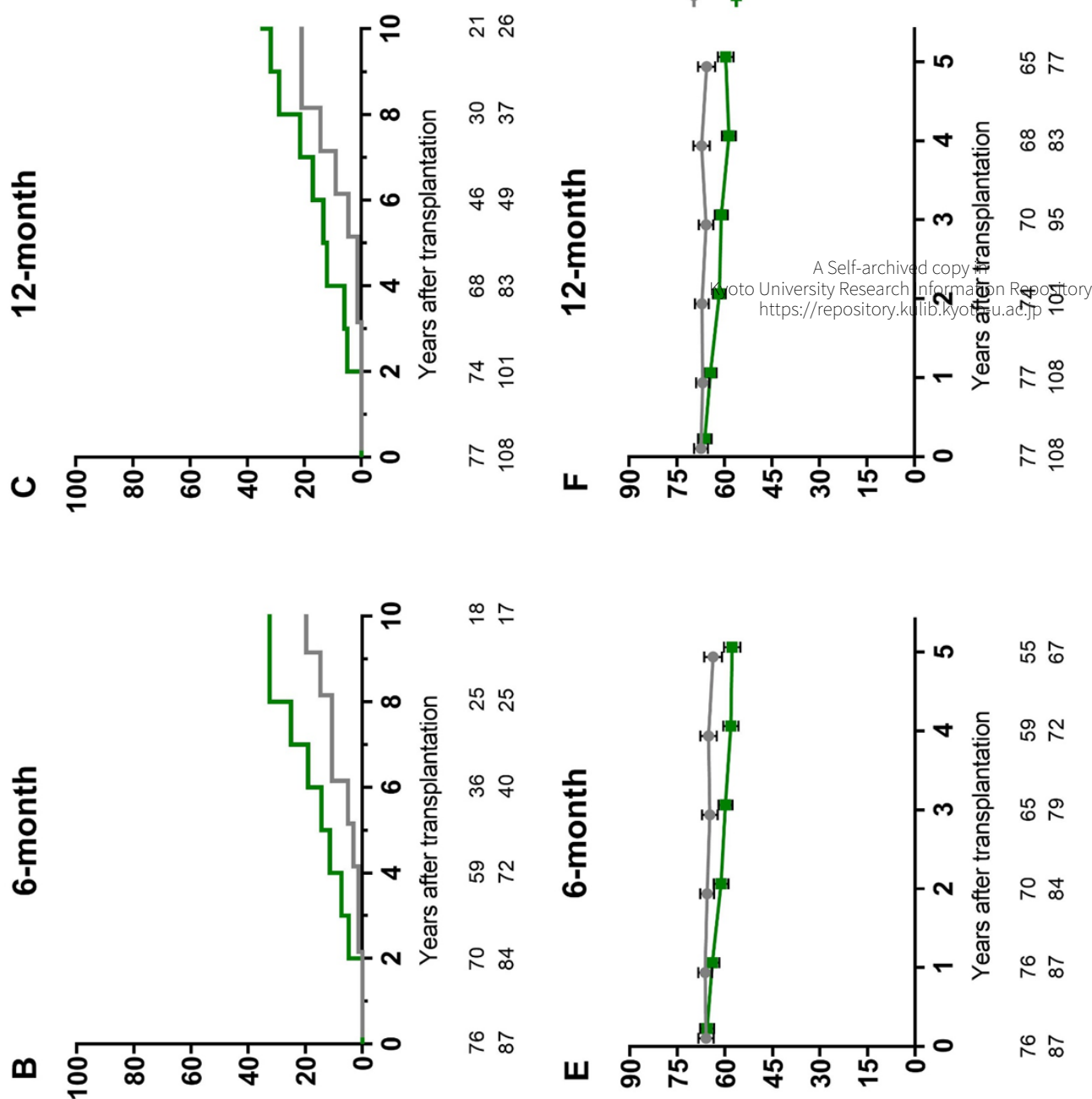

$\stackrel{\infty}{\leftarrow}$
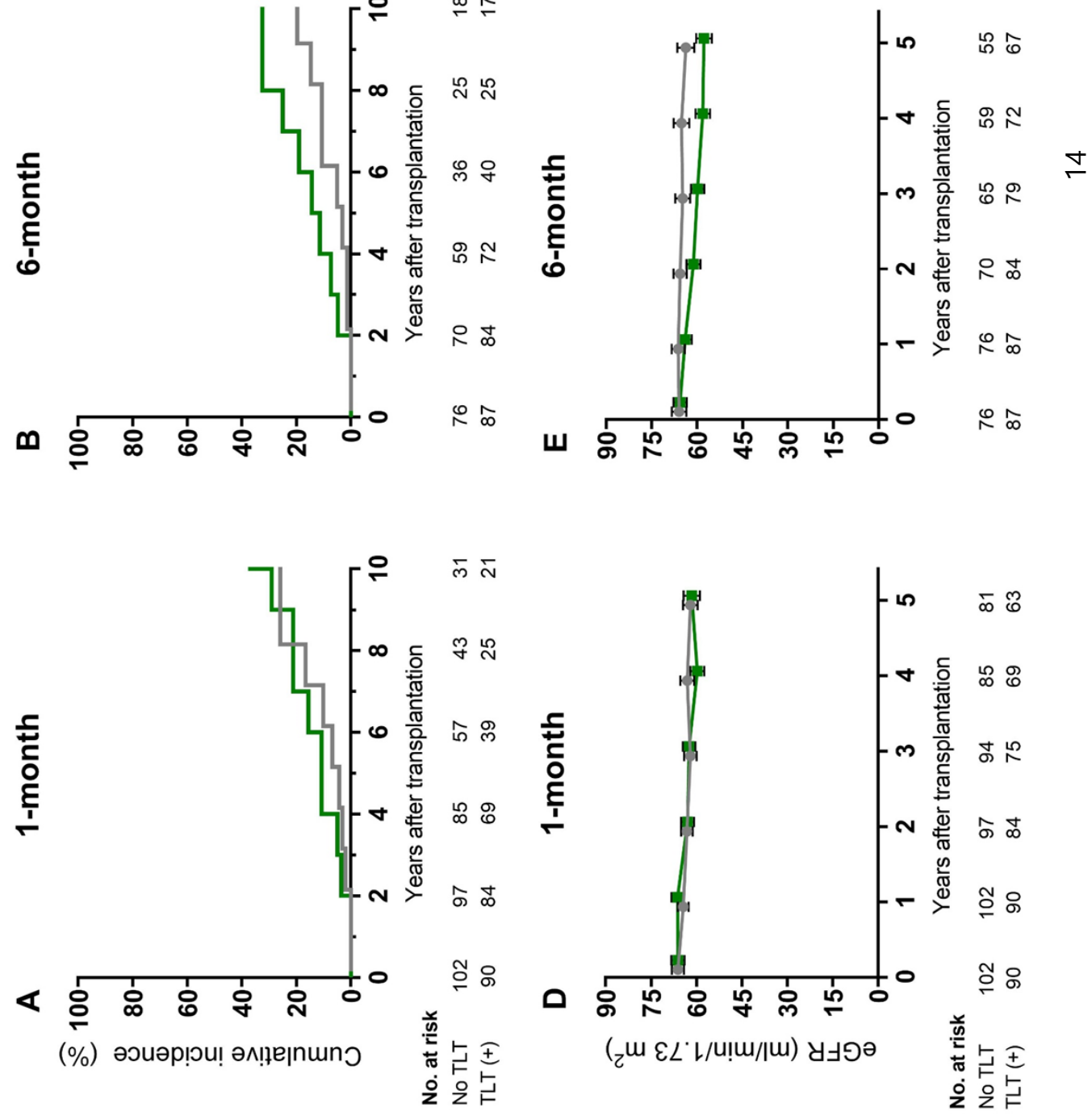


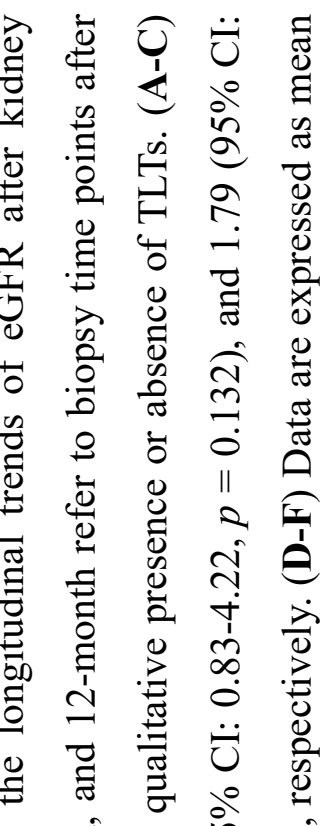



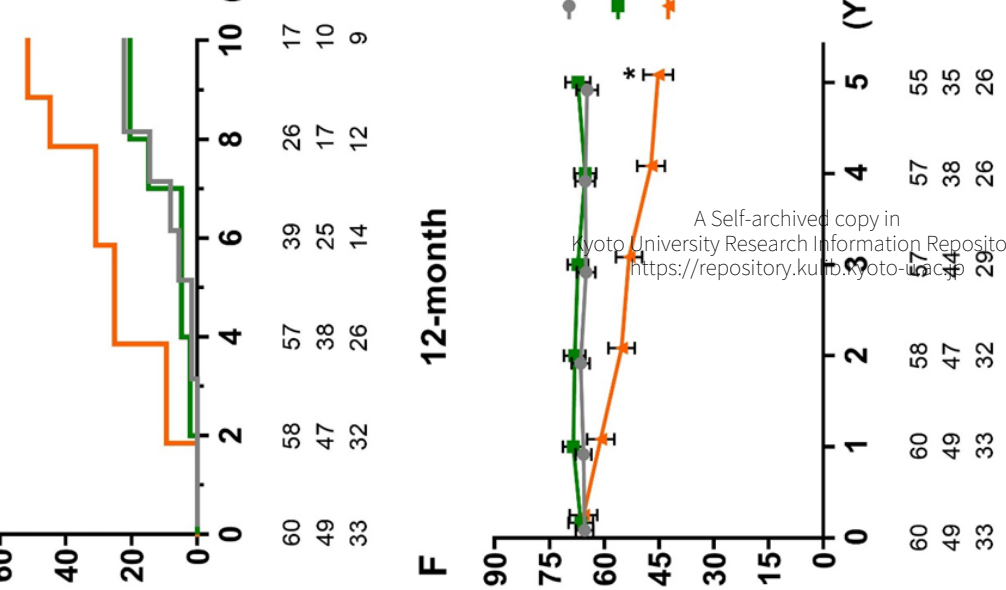

u

ํㅗำ

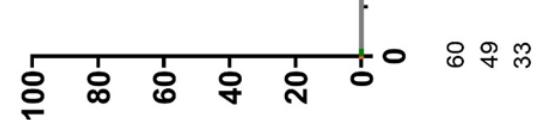

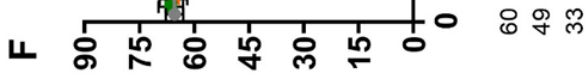
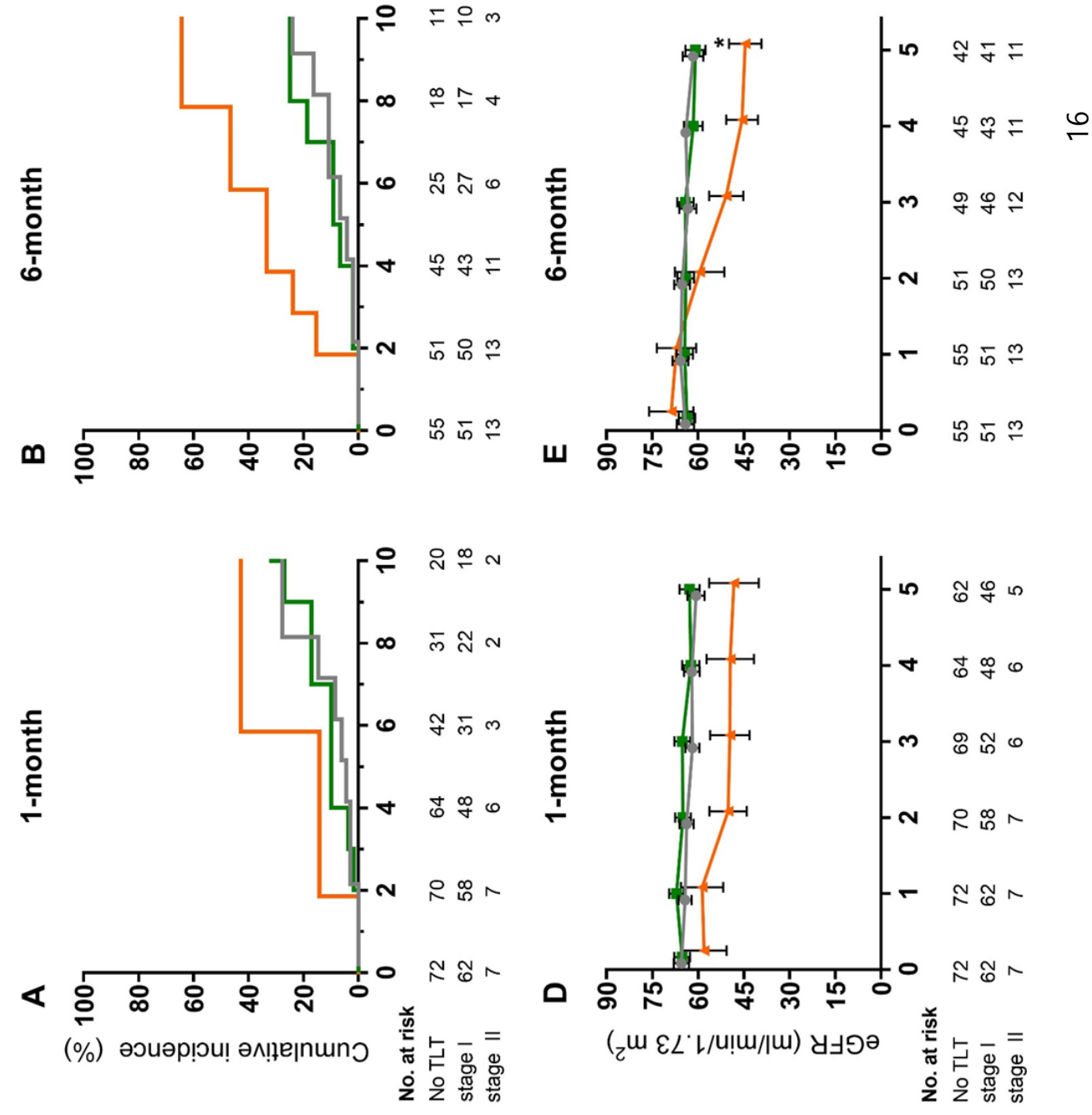


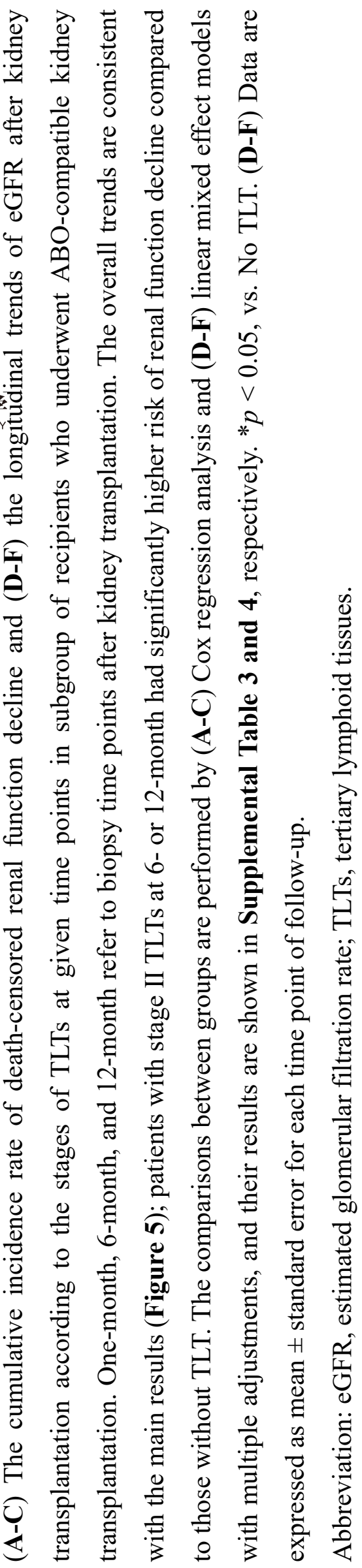

Kyoto University Research Information Repository 
Supplemental Figure 5. The prevalence of donor-specific antibody at 12 months after kidney transplantation according to the stages of tertiary lymphoid tissues

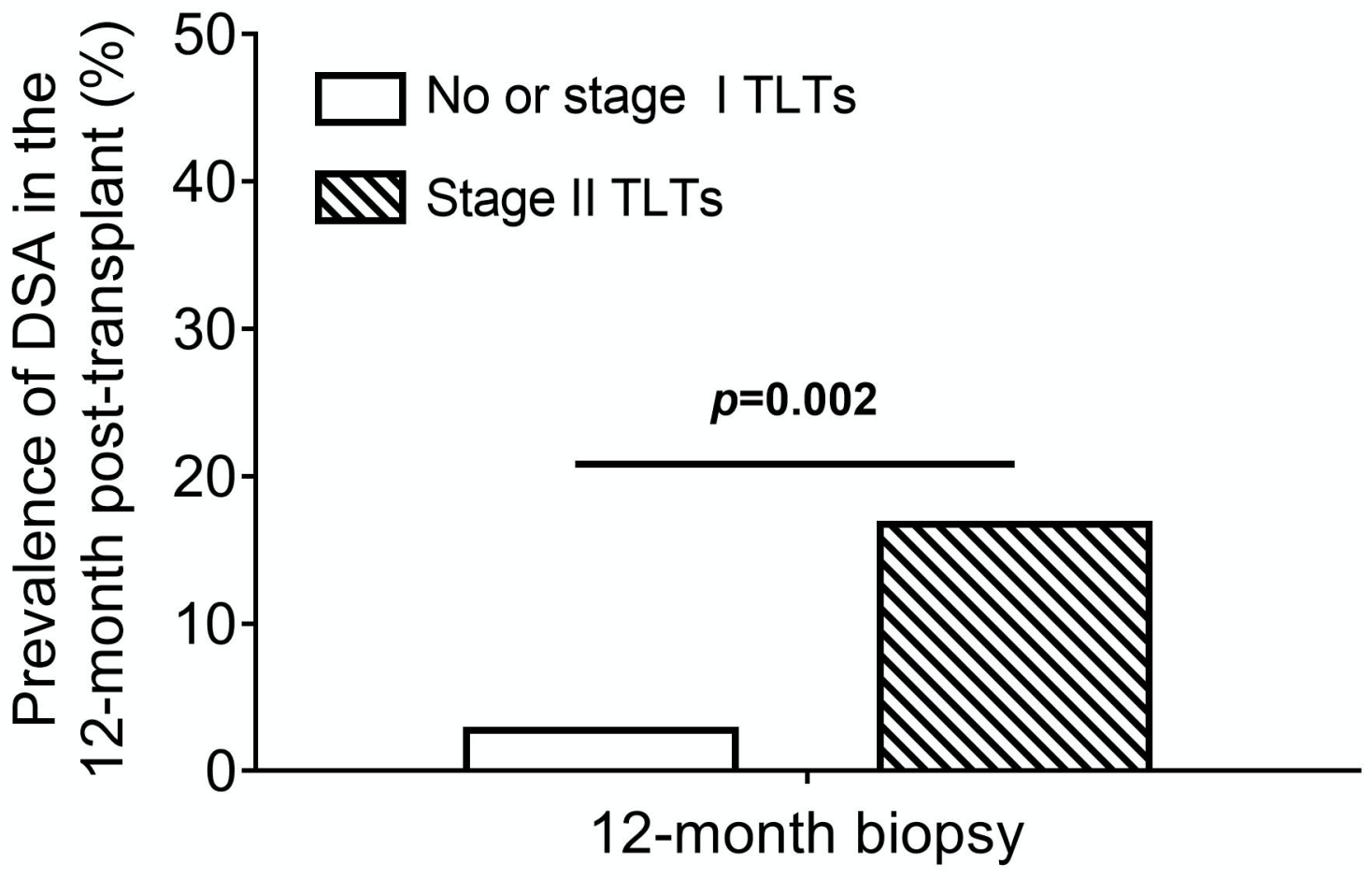

Patients with stage II TLTs at 12-month biopsies more frequently exhibited donor-specific antibodies at 12 months post-transplantation than those with no or stage I TLTs $(p=0.002$ by chi-square test).

Abbreviation: DSA, donor-specific antibody; TLTs, tertiary lymphoid tissues. 


\begin{abstract}
Supplemental Figure 6. Longitudinal trends of renal allograft function according to the history of borderline $T$ cell-mediated rejection within the first year after kidney transplantation
\end{abstract}

\title{
Trends of eGFR
}

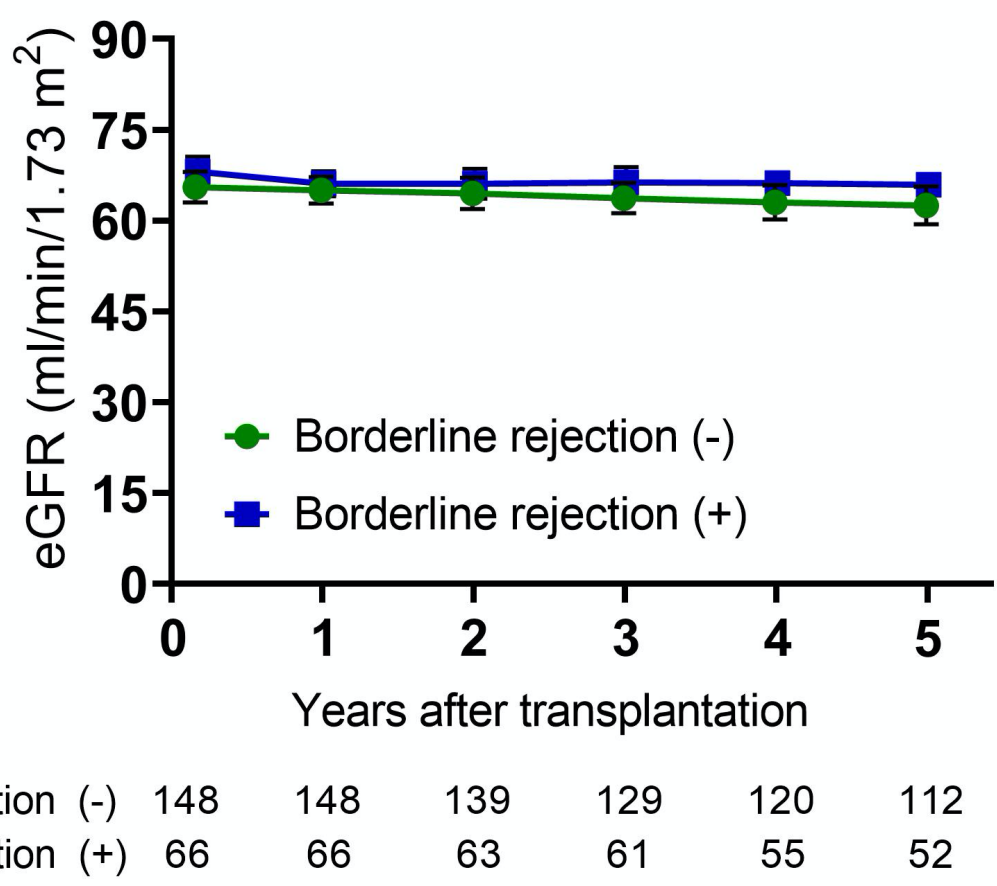

No. at risk

$\begin{array}{lllllll}\text { Borderline rejection (-) } & 148 & 148 & 139 & 129 & 120 & 112\end{array}$

Borderline rejection (+) $66 \quad 66 \quad 63 \quad 61 \quad 55 \quad 52$

A total of 66 kidney transplantation recipients were diagnosed with borderline $\mathrm{T}$ cell-mediated rejection within the first year of post-transplantation and most of them $(63 / 66,95.5 \%)$ were treated with steroid pulse therapy. These patients showed similar levels of graft function over the next 5 years after transplantation compared to those without borderline rejection. Data are expressed as mean \pm standard error for each time point of follow-up.

Abbreviation: eGFR, estimated glomerular filtration rate. 
Supplemental Figure 7. Longitudinal trends of renal allograft function according to the use of pre-transplantation rituximab and the stages of tertiary lymphoid tissues in the 12month biopsies

\section{Trends of eGFR}

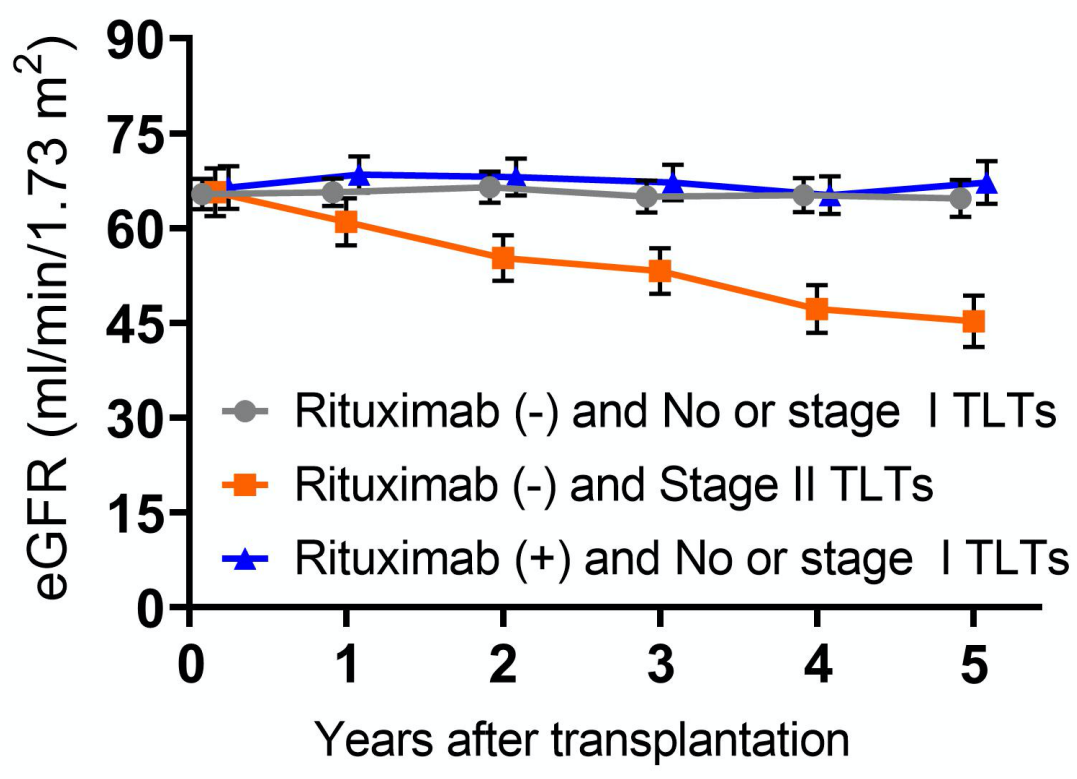

No. at risk

$\begin{array}{lllllll}\text { RTX (-), No/stage I } & 106 & 106 & 102 & 98 & 92 & 87\end{array}$

$\begin{array}{lllllll}\text { RTX (-), stage II } & 32 & 32 & 31 & 28 & 25 & 25\end{array}$

$\begin{array}{lllllll}\text { RTX (+), No/stage I } & 54 & 54 & 50 & 47 & 41 & 35\end{array}$

Patients treated with rituximab before transplantation showed stable graft function over 5 years after transplantation comparable to those with no or stage I TLTs not treated with rituximab. Data are expressed as mean \pm standard error for each time point of follow-up. Note that the trends of eGFR of rituximab-treated patients exhibiting stage II TLTs are not shown because of their small number $(n=3)$.

Abbreviation: eGFR, estimated glomerular filtration rate; TLT, tertiary lymphoid tissue; RTX, rituximab. 


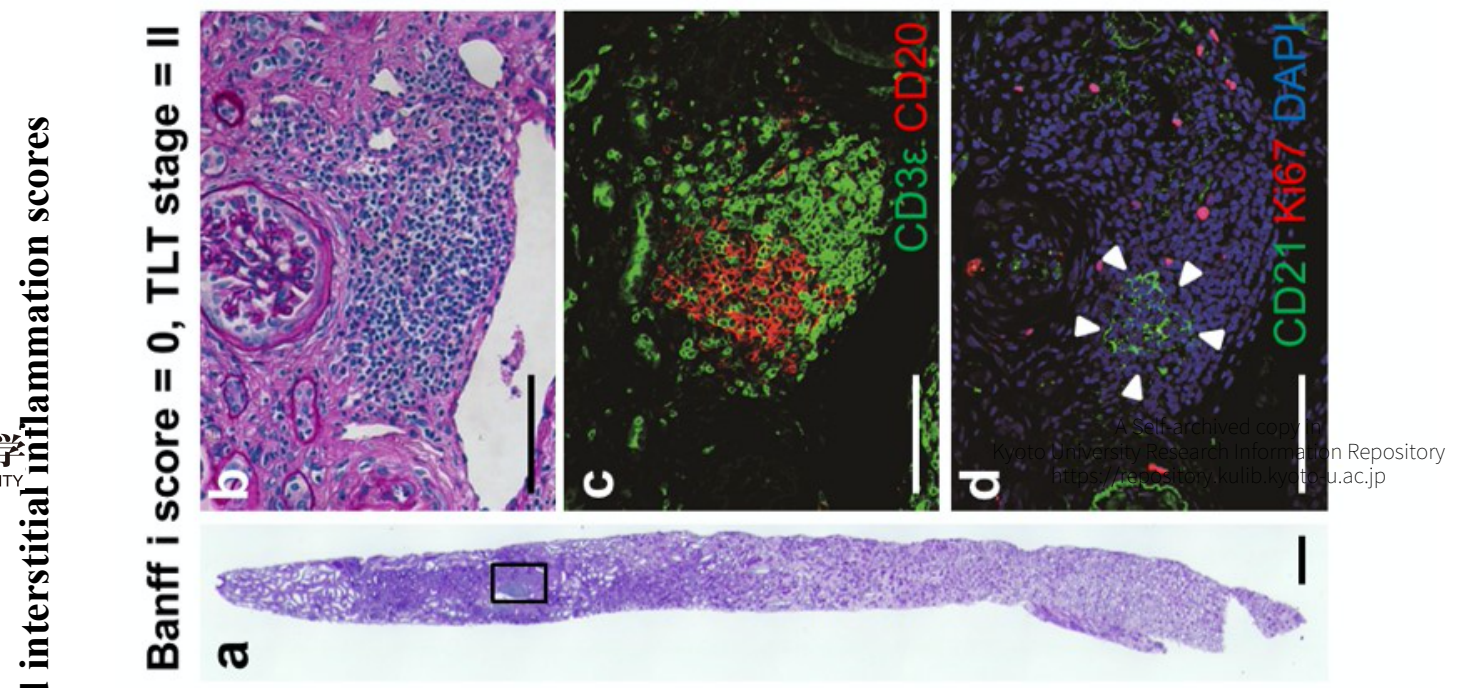

$m$
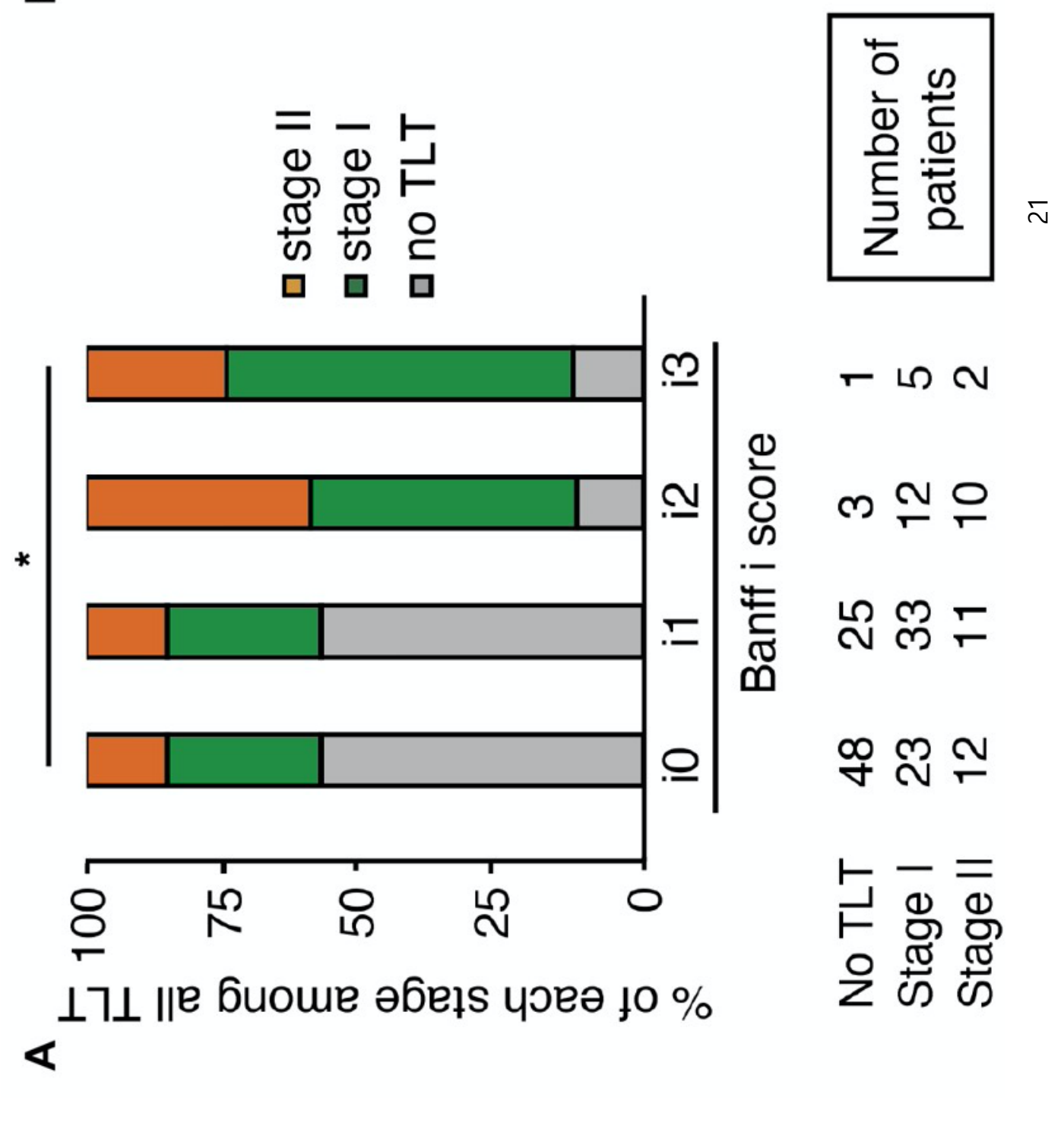
0
0
4
$N$
0
0
0
0

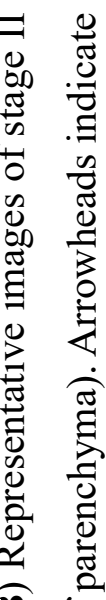

